\title{
WIYN open cluster study
}

\section{Improved kinematic membership and spectroscopy of IC $2391^{\star, \star \star, \star \star \star}$}

\author{
I. Platais ${ }^{1}$, C. Melo ${ }^{2,3}$, J.-C. Mermilliod ${ }^{4}$, V. Kozhurina-Platais ${ }^{5}$, J. P. Fulbright ${ }^{1}$, R. A. Méndez ${ }^{2}$, \\ M. Altmann ${ }^{2}$, and J. Sperauskas ${ }^{6}$
}

\author{
1 Department of Physics and Astronomy, Johns Hopkins University, 3400 North Charles Street, Baltimore, MD 21218, USA \\ e-mail: imants@pha.jhu.edu \\ 2 Departamento de Astronomía, Universidad de Chile, Casilla 36-D, Santiago, Chile \\ 3 European Southern Observatory, Casilla 19001, Santiago 19, Chile \\ ${ }^{4}$ Laboratoire d'Astrophysique de l'École polytechnique fédérale de Lausanne, 1290 Chavannes-des-Bois, Switzerland \\ 5 Space Telescope Science Institute, 3700 San Martin Drive, Baltimore, MD 21218, USA \\ 6 Vilnius University Observatory, Ciurlionio 29, Vilnius 2009, Lithuania
}

Received 2 June 2006 / Accepted 21 August 2006

\section{ABSTRACT}

\begin{abstract}
Context. Young open clusters provide important clues to the interface between the main sequence and pre-main-sequence phases of stellar evolution. The young and nearby open cluster IC 2391 is well-suited to studies of these two evolutionary phases.

Aims. We establish a bona fide set of cluster members and then analyze this set in terms of binary frequency, projected rotational velocities, $[\mathrm{Fe} / \mathrm{H}]$, and lithium abundance. In the wake of the Hipparcos distance controversy for the Pleiades, we compare the mainsequence fitting distance modulus to the Hipparcos mean parallax for IC 2391.

Methods. We have obtained new proper motions for 6991 stars down to $V \sim 13-16$ over a $\sim 9-$ deg $^{2}$ area of the sky comprising

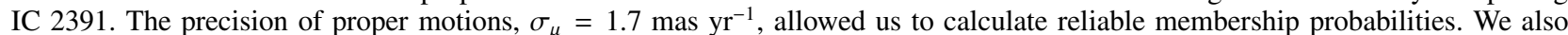
obtained precise radial velocity and $v \sin i$ measurements with CORAVEL and FEROS for 76 probable cluster members. The cluster's mean radial velocity is $+14.8 \pm 0.7 \mathrm{~km} \mathrm{~s}^{-1}$. The FEROS high-resolution spectra were used to determine both the $[\mathrm{Fe} / \mathrm{H}]$ abundance in the four main sequence dwarfs of IC 2391 and the Li abundance in 47 stars. In addition, new BV CCD photometry was obtained for the majority of probable cluster members.

Results. The proper-motion survey covers a 6 times larger sky area than the prior targeted searches for cluster members in IC 2391. A total of 66 stars are considered bona fide cluster members down to a mass equivalent to $0.5 M_{\odot}$. A quarter of them have been newly identified with many in the F2-K5 spectral range, which is crucial for a main-sequence fit. We find a mean $[\mathrm{Fe} / \mathrm{H}]$ value of $+0.06 \pm 0.06$, when a solar abundance of $\log \epsilon(\mathrm{Fe})=7.45$ is adopted. The main sequence fitting yields a distance modulus that is 0.19 mag larger than that derived from Hipparcos parallaxes; thus this offset nearly has the size of a similar offset found for the Pleiades. The Li abundance pattern is similar to the earlier findings and is typical for a $40 \mathrm{Myr}$ old open cluster.

Conclusions. A variety of new data on the probable members of IC 2391 improve essentially all observational parameters of this young open cluster.
\end{abstract}

Key words. open clusters and associations: individual: IC 2391 - astrometry - stars: kinematics - stars: abundances

\section{Introduction}

IC 2391 is a young $(\sim 35 \mathrm{Myr})$ and nearby $(d \sim 150 \mathrm{pc})$ open cluster located in Vela $\left(\ell=270^{\circ}, b=-7^{\circ}\right)$. Its proximity is very appealing for any detailed studies of intrinsically faint low-mass stars and brown dwarfs (Barrado y Navascués et al. 2004). The significance of IC 2391 is clearly demonstrated by a large number of literature references over the last decade in the SIMBAD Astronomical Database: 200 publications where the cluster has been mentioned or studied. One problem facing the researchers of IC 2391 is the scarcity of confirmed cluster

* Based on observations collected at the European Southern Observatory, Chile (Program IDs: 072.D-0107 and 074.D-0096).

$\star \star$ Tables 1, 3, 5, and 6 are only available in electronic form at the CDS via anonymous ftp to cdsarc.u-strasbg.fr (130.79.128.5) or via

http://cdsweb.u-strasbg.fr/cgi-bin/qcat?J/A+A/461/509

$\star \star \star$ Tables 4,8 and 9 are only available in electronic form at

http://www. aanda.org members. For a long time, the known cluster membership was confined to merely $\sim 20$ stars, all brighter than $V \sim 11$ (Hogg 1960). Then, Stauffer et al. (1989) reported on the proper-motion study of 883 stars over a $48^{\prime} \times 41^{\prime}$ area. Proper motions, BVRI photometry, and high-resolution spectroscopy together yielded a list of ten additional probable cluster members down to $V=14$. This list was substantially extended by using the ROSAT imaging data (Patten \& Simon 1993, 1996; Simon \& Patten 1998) to take advantage of the known strong $\mathrm{X}$-ray activity among the young G-K-M spectral type stars. In the follow-up spectroscopic study, Stauffer et al. (1997) confirmed the cluster membership of 23 X-ray selected stars down to $V \sim 15$, using the radial velocity, $\mathrm{Li}$ line, and $\mathrm{H}_{\alpha}$ appearance as membership criteria. Dodd (2004) attempted to identify more cluster members by mining USNO-B and 2MASS catalogs. From 185 astrometrically selected possible cluster members, a total of 35 stars are brighter than $R=15$. However, a disturbingly small fraction of these stars $(\sim 20 \%)$ are common with the Patten \& Simon (1993) list in the same magnitude range and spatial coverage. The latest search 
for cluster members in the central $30^{\prime} \times 30^{\prime}$ region of IC 2391 by the XMM-Newton X-ray observatory resulted in nine relatively faint possible new members (Marino et al. 2005).

As indicated above, proper motions have been used as a kinematic membership discriminator for IC 2391. However, only the study by King (1979) provides precise relative proper motions $\left.\left(\sigma=0.9 \text { mas }^{-1}\right)^{-1}\right)$ down to $V \sim 12$ over a $1.7 \times 0.9$ area. In this study, from a total of 232 stars about 40 have proper motions consistent with membership in IC 2391. No formal membership probabilities are calculated, apparently owing to the sparseness of the cluster. Another way to ascertain the membership status, independent of any assumptions on the astrophysical properties of probable cluster members, is to use radial velocities. There is a rich literature on this subject for IC 2391, e.g., Feinstein (1961), Buscombe (1965), Perry \& Bond (1969), van Hoof (1972), Levato et al. (1988), Stauffer et al. (1997), Barrado y Navascués et al. (1999). Nearly 100 stars have had their radial velocities measured, many of them several times. In many cases, however, the precision of the radial velocities was low, especially for the early and very late type stars, thereby largely precluding assignment of a reliable membership status to them.

Often IC 2391 is considered along with IC 2602, because both have very similar properties and are separated spatially only by $\sim 50 \mathrm{pc}$, thus suggesting a common origin. Their absolute proper motions, however, differ significantly. The projected total velocity in the tangential plane for IC 2391 is 33.8 mas yr $^{-1}$, whereas for IC 2602 it is only 20.5 mas yr$^{-1}$ (Robichon et al. 1999). A much larger tangential velocity of IC 2391 considerably increases the reliability of membership probabilities drawn from proper motions, since a smaller fraction of field stars are expected to share the motion of the cluster. This and the limited precision of our proper motions are one of the main reasons for selecting IC 2391 as the subject of this study.

Randich et al. (2001) spectroscopically analyzed 50 X-ray selected candidate members in IC 2391 and IC 2602. From the analysis of $8 \mathrm{Fe}$ I lines in four stars, the mean metallicity of IC 2391 was derived to be $[\mathrm{Fe} / \mathrm{H}]=-0.03 \pm 0.07$. In this study, $\mathrm{Li}$ abundance was obtained for 32 possible members of IC 2391, covering a wide range of $T_{\text {eff }}-$ from 3500 to $6600 \mathrm{~K}$. It was found that stars warmer than $\sim 5800 \mathrm{~K}$ or more massive than $\sim 1 M_{\odot}$ show no significant signs of Li depletion. For cooler late$\mathrm{G}$ to early-K stars, the pattern of Li abundances in IC 2391 and the Pleiades is similar, although hinting that in this $T_{\text {eff }}$ range $\mathrm{Li}$ is less depleted in IC 2391, as one would expect from the age difference. A more detailed analysis of $\mathrm{Li}$ abundance in IC 2391 is hindered by the small number of stars in the Randich et al. (2001) sample.

Young open clusters appear to have stars with a broad range of rotational rates (Herbst \& Mundt 2005). That is also confirmed by the observed rotational rates for late-type stars (Patten $\&$ Simon 1996) and the $v \sin i$ distribution in IC 2391 (Stauffer et al. 1997). From the standpoint of Li abundances, X-ray luminosities, and stellar evolution, it is vital to identify fast rotators in the enlarged sample of cluster stars.

In some aspects, the level of our understanding of the open cluster IC 2391 is similar to NGC 2451A, which was recently studied by Platais et al. (2001) as one of the WIYN Open Cluster Study (WOCS) targets. The lack of comprehensive astrometric cluster membership prompted us to include IC 2391 among the WOCS clusters. Following the WOCS strategy (Mathieu 2000), we derived new proper motions and calculated the cluster membership probabilities. For many probable cluster members, highresolution spectroscopy served to measure the radial velocities, projected rotational velocities $v \sin i$, Li abundance, and equivalent width of $\mathrm{H}_{\alpha}$. A few carefully selected cluster stars are used to obtain metallicity $[\mathrm{Fe} / \mathrm{H}]$. New CCD photometry is used to construct reliable color-magnitude diagram and perform the isochrone fit.

\section{Astrometric reductions and cluster membership}

A total of four $8 \times 10$ inch photographic plates (scale = $55^{\prime \prime} .1 \mathrm{~mm}^{-1}$ ), taken with the $51 \mathrm{~cm}$ double astrograph of Cesco Observatory in El Leoncito, Argentina, were used for astrometry. Two of these visual-bandpass plates (103a-G emulsion and OG515 filter) were obtained in 1967.29, the other two in 1996.14. An objective wire-grating was used to produce diffraction images for all stars brighter than $V \sim 13$. Each first-epoch plate contains two exposures: a $30 \mathrm{~min}$ and an offset $1 \mathrm{~min}$ exposure.

Our target stars were drawn from the COSMOS/UKST Object Catalog (Yentis et al. 1992). In this catalog the object brightness is given in $B_{\mathrm{J}}$ magnitudes as derived in the natural photographic system (IIa-J emulsion and GG-395 filter) of the UK $1.2 \mathrm{~m}$ Schmidt Telescope at Siding Spring, Australia (Blair $\&$ Gilmore 1982). Due to the scan-time limitations set by the measuring machine, the sample selection required an optimization. All stars down to $B_{\mathrm{J}}=13.0$ were chosen in a $3.5 \times 2.7$ rectangle centered on $\alpha=8^{\mathrm{h}} 40^{\mathrm{m}}$ and $\delta=-52^{\circ} 53^{\prime}$ (equinox $\mathrm{J} 2000.0$ ). A sub-sample of fainter stars at $B_{\mathrm{J}}=14.6$ in the same area served as anonymous astrometric reference stars. Then, within this rectangle all additional stars down to $B_{\mathrm{J}}=16.2$ were selected in a circle with the radius of 0.8 centered on $\alpha=8^{\mathrm{h}} 42^{\mathrm{m}} .5$ and $\delta=-53^{\circ}$.

Altogether, our initial sample included over 7000 stars. All measurable images of these stars were digitized with the Yale 2020G PDS microdensitometer in a fine-raster, object-by-object mode. The image positions were determined using the Yale Image Centering routine (Lee \& van Altena 1983), which includes a two-dimensional Gaussian fit.

The positions and proper motions were calculated using the standard SPM (Southern Proper Motion program) astrometric reductions, described in detail by Girard et al. (1998) and Platais et al. (1998). Owing to the relatively small 9- $\mathrm{deg}^{2}$ field, only linear and quadratic plate-tilt terms were used in the proper-motion plate model. The standard error of proper motions was estimated

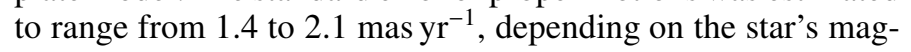
nitude and hence on the number of available grating images. The calculated relative proper motions are free of apparent systematic errors. The distribution of proper motions or a vector-point diagram (VPD) is shown in Fig. 1. A visible clumping of proper

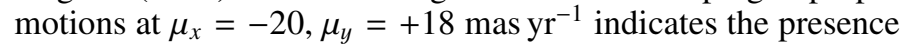
of IC 2391 members.

The local sample method (Kozhurina-Platais et al. 1995) was used to calculate the cluster membership. In this method, for each target star a representative sub-sample (a bin) of other stars is formed that shares the properties of the target, such as the brightness. We used a wide 10-mag sliding brightness bin, which for the brightest and faintest stars narrows down to 5 mag. No spatial window was used for this sample of proper motions. Similar to the case of NGC 2451A (Platais et al. 2001), a flat distribution of field stars in VPD was adopted in the vicinity of the cluster centroid. The resulting membership probability, $P_{\mu}$, is defined as

$P_{\mu}=\frac{\Phi_{\mathrm{c}}}{\Phi_{\mathrm{c}}+\Phi_{\mathrm{f}}}$, 


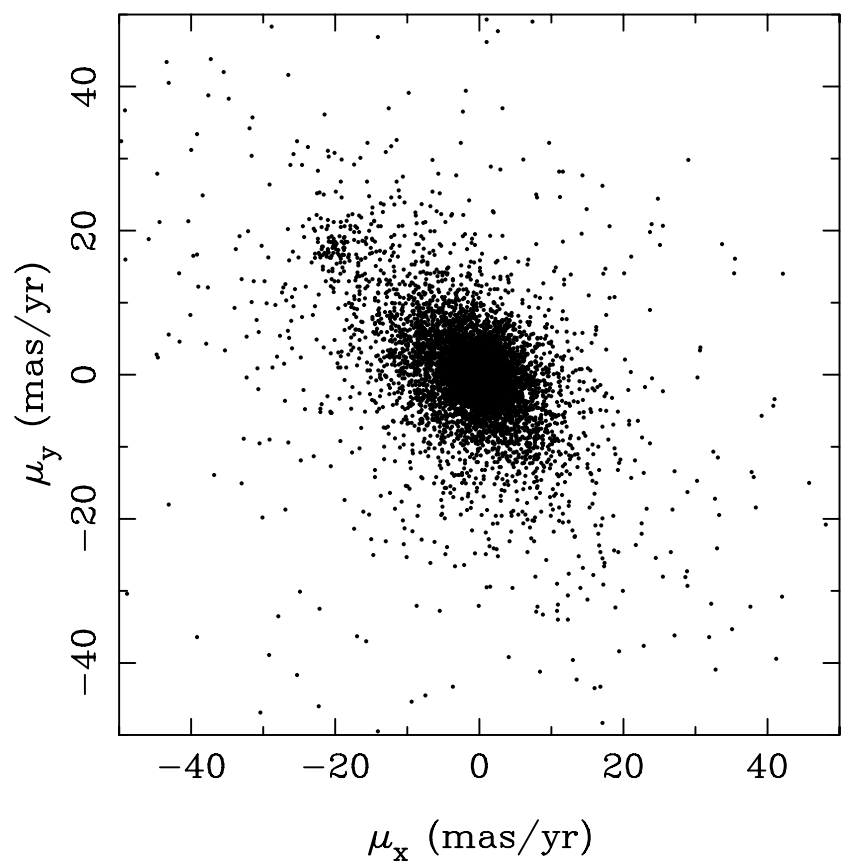

Fig. 1. Proper-motion vector point diagram in the area of IC 2391. A clump of proper motions at $\mu_{x}=-20.1$ and $\mu_{y}=+17.6$ mas $\mathrm{yr}^{-1}$ indicates the location of the cluster.

where, $\Phi_{\mathrm{c}}$ is a Gaussian representing the cluster star distribution in the VPD and $\Phi_{\mathrm{f}}$ is the distribution of field stars, both defined within the same magnitude bin. The following parameters were adopted for the cluster star distribution: the cluster center in the VPD at $\mu_{x}^{\mathrm{c}}=-20.1$ and $\mu_{y}^{\mathrm{c}}=+17.6$ mas $\mathrm{yr}^{-1}$; the Gaussian

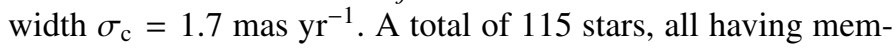
bership probability $P_{\mu} \geq 5 \%$, are listed in Table 1 . A formal sum of membership probabilities indicates that in our sample of 6991 stars, only $\sim 65$ are members of IC 2391.

Finally, precise equatorial coordinates were calculated for all stars, choosing UCAC2 stars (Zacharias et al. 2004) as a reference frame. The coordinate transformation required a quadratic plate model supplemented with two main cubic distortion terms. The standard error of that transformation via the least-squares formalism is $\sim 60$ mas. At the epoch and equinox of J2000, the estimated average accuracy of the catalog ${ }^{1}$ positions is about 30 mas. We note that this study provides precise coordinates for many X-ray selected cluster members, which so far have had only approximate coordinates from Patten \& Simon (1996) and Marino et al. (2005).

\section{Photometry and CCD reductions}

A few sources providing $U B V$ photometry cover mainly the inner area of IC 2391, e.g., Hogg (1960), Lyngå (1960), Perry \& Hill (1969). In January 1997 we obtained new CCD BV photometry for the majority of the possible proper-motion members. The observations were made at the Cerro Tololo Inter-American Observatory (CTIO) $0.9 \mathrm{~m}$ telescope with the Tektronics $2048 \times$ 2048 CCD chip, which covers $13.5 \times 13.5$ on the sky.

As in the case of NGC 2451A (Platais et al. 2001), each probable cluster member or else a group of members with mutual separations less than $\sim 10^{\prime}$ was observed individually. Only a small fraction of these stars could be identified on more

1 The catalog is available at http: //www . astro.yale.edu/astrom/
Table 2. Comparison of $B V$ photometry "CCD-Source".

\begin{tabular}{lccc}
\hline \hline Source & $n$ & $\Delta V$ & $\Delta(B-V)$ \\
\hline Hogg (1960) & 24 & $-0.025 \pm 0.07$ & $+0.016 \pm 0.03$ \\
Lyngå (1960) & 24 & $+0.040 \pm 0.06$ & $-0.011 \pm 0.03$ \\
Perry \& Hill (1969) & 21 & $+0.013 \pm 0.05$ & $+0.012 \pm 0.04$ \\
Forbes et al. (1998) & 19 & $+0.038 \pm 0.06$ & $\ldots$ \\
Eggen (1972) & 11 & $+0.050 \pm 0.06$ & $-0.010 \pm 0.02$ \\
Stauffer et al. (1989) & 5 & $+0.058 \pm 0.05$ & $+0.046 \pm 0.04$ \\
Rollestone \& Byrne (1997) & 4 & $+0.027 \pm 0.02$ & $+0.14 \pm 0.03$ \\
\hline
\end{tabular}

than one CCD frame in each filter. In total, 114 CCD frames were obtained in $B$ and $V$ filters with exposure time varied from 1 to $150 \mathrm{~s}$, depending on the star's magnitude. A set of Landolt (1992) standards was taken three times a night over 22-24 January, 1997 - spanning our observations of IC 2391. The twilight sky frames were used to correct the pixel-to-pixel sensitivity variations.

All CCD frames were reduced using the IRAF DAOPHOTX photometry package. The aperture photometry routine PHOT was applied because the target stars were optimally exposed and well-isolated. The details of transformation of the instrumental magnitudes into the standard $B V$ system for this observing run are given in Platais et al. (2001). The final $B V$ magnitudes are believed to be on the standard system to within 0.02 mag and have a standard error of 0.03 mag in $V$ and 0.02 in $B-V$. It should be noted that the reddest stars may have a slightly less accurate photometry since the reddest standard star has only $B-V=1.18$.

Table 2 shows the comparison of our CCD $B V$ photometry with seven other sources of $B V$ photometry, mainly photoelectric. This table contains the literature reference, the number of common stars $(n)$, mean $\Delta V$ and $\Delta(B-V)$ in the sense of our CCD photometry minus the published one. The errors are standard deviations from the mean calculated difference in magnitude or color. All the sources of photometry are consistent, though on average our CCD $V$-magnitudes appear to be fainter by $\sim 0.03$ than those from the other sources.

\section{CORAVEL radial velocities and $v \sin i$}

The radial-velocity observations were made with the photoelectric spectrometer CORAVEL (Baranne et al. 1979; Mayor 1985) on the Danish $1.54 \mathrm{~m}$ telescope at ESO, La Silla, Chile. They were obtained starting in March 1984 through April 1996 (when the CORAVEL was retired) during the course of regular ESO and Danish time runs allocated to the open cluster studies. In the CORAVEL-type instruments, the spectrum of a star is electromechanically correlated, i.e., scanned with an appropriate spectral mask in the focal plane. The output correlation profile can be described with a Gaussian (position, depth, width) plus a continuum level, readily providing an estimate of radial velocity and $v \sin i$. The latter is computed following the techniques described by Benz \& Mayor (1981, 1984). The radial velocities are on the system defined by Udry et al. (1999), calibrated with high-precision data from the ELODIE spectrograph (Baranne et al. 1996).

The initial sample consisted of only 8 stars, all brighter than $B=11.6$ and already thought to be possible members in 1983 . Three of them $(3664=$ SHJM 7, 3722, 5382) were found to be SB1 spectroscopic binaries and two were found to be SB2 (389, 4413). It should be noted that CORAVEL can detect a secondary if the magnitude difference is smaller than $\sim 1.5-1.8$ mag. 
The list of candidate cluster members was greatly enlarged by the ROSAT X-ray detections in the area of IC 2391 (Patten $\&$ Simon 1993, 1996). A total of 17 such stars $(B<13.8)$ were observed once or twice in February 1995 and/or January-April 1996. Among them, two additional likely spectroscopic binaries $(5768,5859)$ were detected.

Two presumably constant stars are common to the CORAVEL and FEROS (see Sect. 5) samples. The radial velocity difference, in the sense CORAVEL-FEROS, for star 4362 is $+0.09 \mathrm{~km} \mathrm{~s}^{-1}$ and for star 4809 is $-0.10 \mathrm{~km} \mathrm{~s}^{-1}$. This indicates very good agreement between the two systems. There are 13 stars in common with the radial-velocity data obtained in 1995 at CTIO by Stauffer et al. (1997). One of them, 5859=VXR 67a, appears to be a spectroscopic binary judging from two CORAVEL observations (Table 3 ). The spectroscopic binary, suspected by Stauffer et al. (1997), 4549=VXR 30, is a definite SB1 from the CORAVEL data. For the remaining 11 stars, the radial velocity difference "CORAVEL-CTIO" is $-0.6 \pm 0.3 \mathrm{~km} \mathrm{~s}^{-1}$, which is in good agreement with the listed internal and external errors by Stauffer et al. (1997).

Table 3 contains all CORAVEL heliocentric radial velocities and their estimated standard errors. The last four entries were obtained with the Lithuanian CORAVEL-type spectrometer (Upgren et al. 2002) at the CASLEO $2.2 \mathrm{~m}$ telescope in El Leoncito, Argentina, in February 2002.

Table 4 lists the mean radial velocities, $v \sin i$, the associated errors and other parameters. For a star, depending on the structure of the correlation profile, up to three sets of parameters (for components A and B, and a blend) can be given. The estimated formal standard deviation of the mean radial velocity is denoted by $\sigma$. The estimated mean internal uncertainty of a radial velocity measurement is denoted by $\epsilon=\max (\sigma$, mean error $) / \sqrt{n}$, where $n$ is the number of measurements. The internal error of an individual observation consists of three components as described by Baranne et al. (1979) and Mermilliod \& Mayor (1989). We note that the uncertainty, $\epsilon$, is progressively underestimated at increasing $v \sin i$ values (Nordstroem et al. 1996). The ratio, standard deviation $\sigma$ vs. the mean internal uncertainty of individual measurements, denoted by the $\mathrm{E} / \mathrm{I}$, is used to calculate the probability $P\left(\chi^{2}\right)$ that the scatter is due to random noise. A star is considered a spectroscopic binary, if $P\left(\chi^{2}\right) \leq 0.001$. In the case of a single measurement, the $P\left(\chi^{2}\right)$ is meaningless and marked by 9.999 . Additional Cols. $6-9$ contain the number of measurements, $n$, the time span in days covered by the observations, $\Delta T$, measured $v \sin i$ and its standard error. Binarity status and the component are indicated in last column.

Finally, we note that the systems of $v \sin i$ from CORAVEL, FEROS, and Randich et al. (2001) are all in excellent agreement for $v \sin i \leq 20 \mathrm{~km} \mathrm{~s}^{-1}$ to within $\sim 1 \mathrm{~km} \mathrm{~s}^{-1}$, but can differ by up to $\sim 10 \mathrm{~km} \mathrm{~s}^{-1}$ for large $v \sin i$ and/or in some cases of spectroscopic binaries.

\section{Spectroscopy with FEROS}

The spectroscopic observations were carried out in two observing runs in February 2004 and February 2005 using the highresolution, two-fiber $(R \sim 50000)$ FEROS echelle spectrograph (Kaufer et al. 1999) at the ESO $2.2 \mathrm{~m}$ telescope in La Silla, Chile. This spectrograph provides a wavelength range from 360 to $920 \mathrm{~nm}$, covered by 39 echelle orders. Such a wide wavelength range is essential in the chemical abundance studies allowing one to select appropriate unblended metal lines. The observations were taken in the Object-Sky mode in which the target is centered onto the Object fiber, whereas the Sky fiber

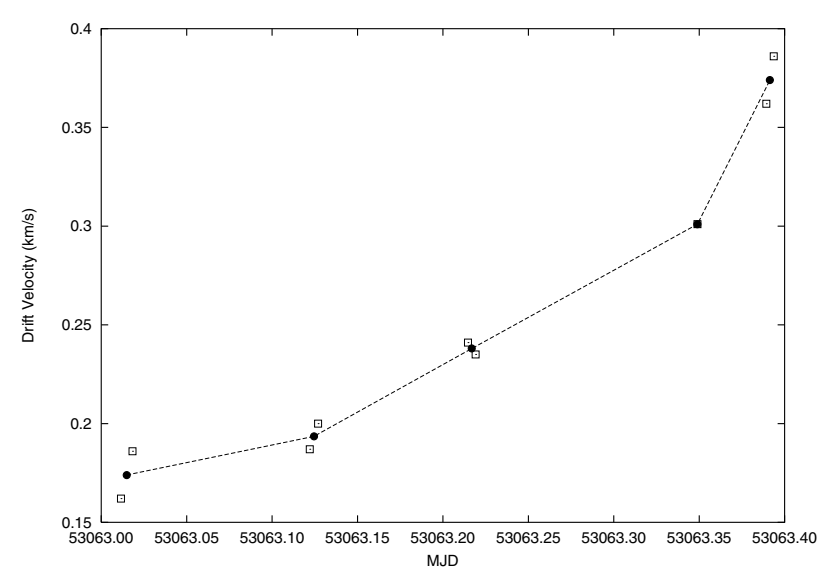

Fig. 2. Drift correction for the night of February 27, 2004. Standard stars are shown as open squares and the mean drift as filled circles. The dashed line is the adopted drift as a function of time.

collects the light from the sky background. The reductions of spectra were performed using the standard FEROS pipeline, which includes flat-fielding, sky-background subtraction, removal of cosmic rays, wavelength calibration, and barycentric velocity correction. The pipeline yields a $1 D$ re-binned spectrum evenly sampled at $0.03 \AA$ A steps.

Radial velocities are derived by cross-correlation techniques using a K0 III spectral type digital binary mask as the template (Baranne et al. 1979; Queloz 1995). The resulting crosscorrelation function (CCF) can in most cases be approximated by a Gaussian function whose center readily gives the radial velocity and the width (Gaussian $\sigma$ ) related to broadening mechanisms such as turbulent motion, gravity pressure, and rotation.

Owing to a relatively high $S / N$, normally higher than 50 (see Table 5), the photon noise errors in our radial velocity measurements typically range between $5-15 \mathrm{~m} \mathrm{~s}^{-1}$. The final uncertainties in derived radial velocities are a combination of the photon noise errors and the overnight spectrograph drift due to the changes in the index of air refractivity and atmospheric pressure. Usually, these shifts are on the order of a few hundred $\mathrm{m} \mathrm{s}^{-1}$ per night. One way to correct for this drift is to have the calibration lamp illuminate a second fiber while the first fiber receives the stellar light. Although the FEROS two-fiber configuration allows for this option (Setiawan et al. 2000), the subsequent data reduction and analysis is complicated and not warranted for young stars where the radial-velocity jitter exceeds the internal precision of measurement by a lot. For example, for very active and young $\mathrm{T}$ Tauri type stars, this jitter is not lower than $\sim 0.6 \mathrm{~km} \mathrm{~s}^{-1}$ and can be as high as $\sim 2 \mathrm{~km} \mathrm{~s}^{-1}$ (Melo 2003). With age these effects gradually abate, though the rotational modulation of stellar active regions can generate a radial velocity scatter up to $50 \mathrm{~m} \mathrm{~s}^{-1}$ even at the age of Hyades at $\sim 600 \mathrm{Myr}$ (Paulson et al. 2004). We therefore opted for a simpler approach, as described below.

In order to correct for this drift, 1-3 radial velocity standards from the CORALIE extra-solar planet survey were observed a few times during the night. For each standard star, the velocity drift was computed as the difference between the CORALIE radial velocity and the observed radial velocity. In the case of more than one standard star, the mean drift was calculated. The radial-velocity corrections for the program stars were computed using a linear interpolation between each two drift points. A typical drift correction is shown in Fig. 2. Over two observing runs, the rms of the differences between the corrected radial velocities 
of standard stars and the CORALIE radial velocities is below $20 \mathrm{~m} \mathrm{~s}^{-1}$, indicating that the procedure works well. One should keep in mind, however, that the final uncertainty of radial velocities could be higher, since at some level a linear interpolation might not approximate the actual pattern of a drift accurately. We note that the rms scatter of the drift correction between the FEROS and CORALIE radial velocities is $\sim 150 \mathrm{~m} \mathrm{~s}^{-1}$, similar to the value found by Melo et al. (2001) using $\tau$ Cet as a standard.

A few double-lined spectroscopic binaries were found among our target stars (Table 5, Fig. 5). Their cluster membership status is briefly discussed in Sect. 6.4.

\subsection{FEROS $v \sin i$}

The width, $\sigma$, of the cross-correlation function (CCF) is a product of several broadening mechanisms related to gravity, turbulence, magnetic fields, effective temperature, metallicity, and rotation. In addition, the instrumental profile also contributes to the broadening of spectral lines and, therefore, to the CCF. Thus, in order to correctly measure the contribution of rotation to the width of the CCF, we should model all sources of broadening, except what is due to rotation. The width of FEROS CCF was calibrated as described in Melo et al. (2001). For fast rotators $\left(v \sin i \gtrsim 30 \mathrm{~km} \mathrm{~s}^{-1}\right)$, the final $v \sin i$ was derived as follows. The CCF is fitted by a family of functions $C C F_{v \sin i}=$ $C-D\left[g_{0} \otimes G(v \sin i)\right]$ which is a convolution of the CCF of a non-rotating star, $g_{0}$, in turn approximated by a Gaussian and the Gray (1992) rotational profile computed for a set of discrete rotational velocities, $G(v \sin i)$. For each function $C C F_{v \sin i}$, we can find the radial velocity $V_{\mathrm{r}}$, the depth $D$, and the continuum $C$ by minimizing the quantity $\chi_{v \sin i}^{2}$. Figure 1 in Melo (2003) illustrates this method. The uncertainty of the $v \sin i$ measurement is $\sim 1.5 \mathrm{~km} \mathrm{~s}^{-1}$ for $v \sin \lesssim 30 \mathrm{~km} \mathrm{~s}^{-1}$ and $\sim 10 \%$ of the $v \sin i$ value for $v \sin \gtrsim 30 \mathrm{~km} \mathrm{~s}^{-1}$. The FEROS measurements of $v \sin i$ are listed in Table 5. The averaged $v \sin i$ values are given in Table 8 . In the case of SB2 spectroscopic binaries, an average $v \sin i$ is given only for the A-component.

\section{2. $[\mathrm{Fe} / \mathrm{H}]$ determination}

The FEROS high-resolution spectra are used to determine the $[\mathrm{Fe} / \mathrm{H}]$ abundance of IC 2391. However, other abundance studies of relatively young open clusters, such as the Hyades, Pleiades, and the Ursa Major moving group (Schuler et al. 2006; Yong et al. 2004; Paulson et al. 2003), have found that the atmospheres of the G- and K-dwarfs show deviations from the simple, plane-parallel atmosphere models used in most abundance studies. High excitation lines and lines of ionized species are stronger than predicted by simple model atmospheres, even though the same models perform adequately for older G- and K- dwarfs like the Sun. Schuler et al. (2006) suggest that photospheric spots and faculae in young dwarfs can possibly produce such deviations.

Until the solution to this problem is found, we only perform a simple abundance analysis on a few stars in IC 2391. The atmosphere's problem is minimized in warmer G-dwarfs, hence we chose four slowly-rotating G-dwarfs with $T_{\text {eff }}$ values ranging between $5200 \mathrm{~K}$ and $5900 \mathrm{~K}$ in the sample.

We adopted the list of iron lines from Fulbright et al. (2006). The lines in this list were selected to be those least affected by blending for use in the study of metal-rich bulge giants, where the differential analysis was done relative to Arcturus. For this study, we used the Sun as the differential standard and adopt a solar iron abundance of $\log \epsilon(\mathrm{Fe})=7.45$. The lines were measured manually using the IRAF splot package, and the measured equivalent widths are given in Table 6. We measured the solar line equivalent widths from the Solar Atlas by Kurucz et al. (1984) and a high $S / N(>200)$ FEROS sky spectrum. We did not use lines stronger than $\sim 120 \mathrm{~m} \AA$.

There is good agreement between the results from the Solar Atlas and the FEROS solar spectrum. If the $g f$-values of the Fe lines are adjusted to yield a solar Fe abundance of $\log \epsilon(\mathrm{Fe})=7.45$ for the equivalent widths measured in the Solar Atlas, the FEROS sky spectrum yields an Fe I abundance of $7.44 \pm 0.06$ (97 lines) and an Fe II abundance of $7.42 \pm 0.07$ ( 5 lines). Therefore, we believe that the instrumental effects in our differential analysis method are negligible.

Due to the aforementioned potential problems with the atmospheres, we did not use spectroscopic indicators to set the stellar parameters, with the exception of setting the model atmosphere $[\mathrm{m} / \mathrm{H}]$ value to match the derived $[\mathrm{Fe} / \mathrm{H}]$ value. We used a grid of solar-abundance ratio atmospheres by Fiorella Castelli ${ }^{2}$ that include updated opacity distribution functions and the 2002 version of the MOOG spectrum synthesis program (Sneden 1973).

We set the $T_{\text {eff }}$ values as an average from three color-to- $T_{\text {eff }}$ calibrations for dwarf stars (Ramírez \& Meléndez 2005), adopting the initial $[\mathrm{Fe} / \mathrm{H}]=0$, very close to the estimate by Randich et al. (2001). We used $B-V, V-J$, and $V-K_{s}$ color indices to obtain $T_{\text {eff }}$, where $J$ and $K_{s}$ are the 2MASS magnitudes (Skrutskie et al. 2006). In the temperature range 4400-5700 K, the common stars between our study and Randich et al. (2001) indicate a nearly perfect match of temperature scales. If all nine common stars are considered, the rms scatter of differences approaches $140 \mathrm{~K}$. It should not be overlooked, however, that such photometrically-calibrated effective temperatures may be biased (e.g., cooler), if a star is a binary and/or still on the pre-mainsequence evolutionary tracks. The Ramírez \& Meléndez (2005) calibrations do not consider such effects though their presence in IC 2391 is undeniable (see Sect. 6.5). We derived the surface gravities $\log g$ by interpolation of the 35-Myr, solar-metallicity isochrone by Girardi et al. (2002). Finally, we used the relationship provided by Allende Prieto et al. (2004) to set the microturbulence parameter $v_{\mathrm{t}}$.

The derived atmosphere parameters and Fe abundances are given in Table 7. The line-by-line abundances show slight trends with respect to excitation potential and line strength, but we did not adjust the stellar parameters or remove any high-excitation lines from the analysis. We point out an especially large difference (mean of $0.15 \pm 0.06 \mathrm{dex}$ ) between the abundances derived from Fe I and Fe II lines, noting that by definition these same lines give the same abundance for both species in the Sun. This is similar to what was seen in studies of other young open clusters, so we only used the results for the Fe I lines to derive the mean cluster abundance.

The weighted mean $[\mathrm{Fe} \mathrm{I/H]}$ value for the four IC 2391 stars is $+0.06 \pm 0.06$ (s.d.). When adopting the solar abundance of $\log \epsilon(\mathrm{Fe})=7.45$, the mean $[\mathrm{Fe} / \mathrm{H}]$ reported by Randich et al. (2001) translates into $[\mathrm{Fe} / \mathrm{H}]=+0.04 \pm 0.07$, a metallicity estimate nearly identical to our value. Recently, in the framework of UVES Paranal Observatory Project, Stütz et al. (2006) obtained elemental abundances for five early-type stars in IC 2391. The weighted mean $[\mathrm{Fe} / \mathrm{H}]$ value for the two bona fide cluster members (HD 74275 = 4522 and SHJM $2=3722$ ) from this study is $+0.10 \pm 0.07$, again in good agreement with our value of $[\mathrm{Fe} / \mathrm{H}]$. A third bona fide member HD $74044=7027$ was also included in the Stuitz et al. (2006) analysis; however, it shows signs of

\footnotetext{
2 http://wwwuser.oat.ts.astro.it/castelli/
} 
Table 7. Derived stellar atmosphere parameters and Fe abundance.

\begin{tabular}{rrrrrrrrrrrrr}
\hline \hline Star & $\begin{array}{c}T_{\text {eff }} \\
\left({ }^{\circ} \mathrm{K}\right)\end{array}$ & $\log g$ & {$[m / \mathrm{H}]^{a}$} & $v_{\mathrm{t}}$ & $\log \epsilon(\mathrm{Fe} \mathrm{I})$ & $\sigma$ & $N$ & $\log \epsilon(\mathrm{Fe} \mathrm{II})$ & $\sigma$ & $N$ \\
& & & $\left(\mathrm{~km} \mathrm{~s}^{-1}\right)$ & & & & \\
\hline 665 & 5451 & 4.53 & +0.05 & 1.25 & 7.50 & 0.06 & 64 & 7.60 & 0.07 & 5 \\
1560 & 5830 & 4.48 & +0.13 & 1.38 & 7.58 & 0.07 & 76 & 7.87 & 0.03 & 5 \\
3359 & 5280 & 4.55 & -0.01 & 1.17 & 7.44 & 0.06 & 73 & 7.64 & 0.06 & 4 \\
4362 & 5616 & 4.51 & +0.05 & 1.30 & 7.50 & 0.07 & 67 & 7.59 & 0.04 & 5 \\
\hline
\end{tabular}

${ }^{a}$ Input model atmosphere abundance on a scale where the solar $\log \epsilon(\mathrm{Fe})=7.45$.

being chemically peculiar and, hence, is not suitable for deriving average elemental abundances in cluster stars.

Abundances derived from Fe I lines are sensitive to the adopted $T_{\text {eff }}$ values: a $+100 \mathrm{~K}$ increase in $T_{\text {eff }}$ will raise the $[\mathrm{Fe} / \mathrm{H}]$ value by about +0.10 dex. Our temperature scale comes only from photometric colors, so it is sensitive to errors in the photometry, to color shifts due to unresolved binaries, and to the intrinsic uncertainties of the fitting functions used to derive the $T_{\text {eff }}$ scales (the $\sigma T_{\text {eff }}$ values the $T(B-V), T(V-J)$, and $T(V-K)$ calibrations are $88 \mathrm{~K}, 62 \mathrm{~K}$, and $50 \mathrm{~K}$, respectively). For example, we note that star 3359 is slightly above the isochrone fitting the main sequence in two out of the three color-magnitude diagrams (see Sect. 6.5). If this is due to the problem in photometry, and we have adopted a $T_{\text {eff }}$ value that could be lower than true $T_{\text {eff }}$ by $\sim 50 \mathrm{~K}$, then our final $[\mathrm{Fe} / \mathrm{H}]$ value for star 3359 is too low by $\sim 0.05$ dex.

\subsection{Li abundance}

The equivalent width of $\mathrm{Li} 6708 \AA$ is known to be a good youth indicator for cool stars (late $\mathrm{G}$ to mid-M type stars) whose interiors are fully convective during the pre-main sequence phase (e.g., Martin 1997). For hotter stars (F to mid-G corresponding to $B-V=0.4-0.7)$, the development of a radiative core in the premain-sequence phase prevents a rapid Li depletion in these stars. Thus, for hotter stars, the changes in Li abundance are insignificant between a few Myr and the Pleiades age (e.g., Soderblom et al. 1993).

The equivalent width of $\mathrm{Li} 6708 \AA$ and $\mathrm{H}_{\alpha}$ was measured manually using the IRAF splot task. Because in fast rotating stars this Li feature is blended with an Fe I line at 6707.441 $\AA$, we applied an empirical correction (Sect. 2.2 Soderblom et al. 1993) to the equivalent width of the $\mathrm{Li}$ feature for all stars with $v \sin i>10 \mathrm{~km} \mathrm{~s}^{-1}$. Then, the equivalent width was converted into $\mathrm{Li}$ abundance, $\log N(\mathrm{Li})$, via the grid of curves of growth tabulated by Soderblom et al. (1993). Effective temperatures were derived as described in Sect. 5.2. The initial analysis of the $\log N(\mathrm{Li})$ distribution as a function of $T_{\text {eff }}$ showed a number of stars with abnormally high $\mathrm{Li}$ abundance, exceeding $\log N(\mathrm{Li})=3.5 \mathrm{dex}$. In our FEROS spectra these stars have $v \sin i \geq 40 \mathrm{~km} \mathrm{~s}^{-1}$ and a poorly-defined continuum around the Li feature. For such stars, it was decided to adopt the smoothed adjacent spectrum just outside the expected width of a $\mathrm{Li}$ feature for a continuum. The measured equivalent widths, $E W(\mathrm{Li})$, are listed in Table 8. Ambiguous lithium non-detections due to the high rotational velocity or binarity are marked by -99 . For stars with $v \sin i<15 \mathrm{~km} \mathrm{~s}^{-1}$, the rms scatter of measured $E W \mathrm{~s}$ from two independent measurements is estimated to be $\sim 6 \mathrm{~m} \AA$. In this range of $v \sin i$, there are only three stars, 4362, 4413, and 5859, in common with Randich et al. (2001). The measurements of $E W$ in both studies agree to within $2 \sigma$ of the errors quoted by Randich et al. (2001). For bona fide cluster members

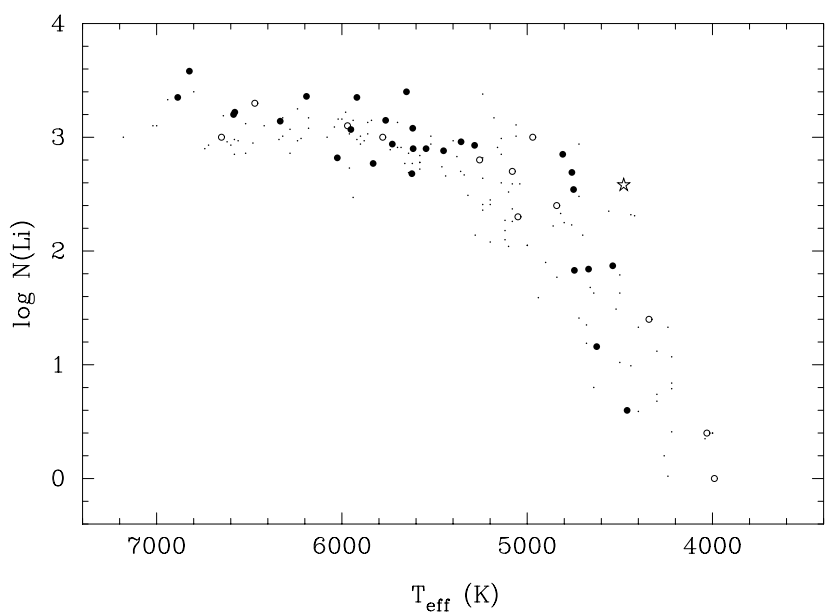

Fig. 3. Lithium abundance as a function of $T_{\text {eff }}$. The bold dots denote bona fide members of IC 2391 from Table 8 . The circles indicate bona fide members having their $\mathrm{Li}$ abundance measured only by Randich et al. (2001). The location of uncertain member $1820=$ SHJM 3 (see Sect. 6.2) is marked by the open star. Superimposed dots represent the Pleiades members from Soderblom et al. (1993). It appears that in the range $4600<T_{\text {eff }}<4800 \mathrm{~K}$ the cluster stars undergo a rapid phase of Li depletion.

of IC 2391, the distribution of lithium abundance as a function of $T_{\text {eff }}$ is shown in Fig. 3.

From the FEROS spectra we also estimated an equivalent width of $\mathrm{H}_{\alpha}$. Eight relatively cool stars in Table 8 show $\mathrm{H}_{\alpha}$ in emission and therefore have negative $E W$ values.

\section{Results}

One of the goals of this paper is to assemble a list of bona fide members of IC 2391 and their basic properties down to $V \sim 15$. Such a list is expected to be useful for subsequent future studies of this sparse open cluster.

\subsection{Bona fide cluster members}

We used the following criteria to decide whether or not to assign the status of bona fide member to a probable cluster member:

1. Proper motion membership probability $P_{\mu} \geq 5 \%$, or all 115 stars from Table 1 . This low $P_{\mu}$ threshold is chosen in order to essentially preserve all possible cluster members.

2. Within the photometric errors a star is on the zero-age main sequence (ZAMS) or above it due to the binarity and/or the pre-main sequence status at $V>12$.

3. The radial velocity is within $\sim 3 \mathrm{~km} \mathrm{~s}^{-1}$ around the cluster's mean $\langle R V\rangle=+14.8 \mathrm{~km} \mathrm{~s}^{-1}$. Since most of our radial velocity measurements were obtained at a single epoch, this limit works against the single-lined binary cluster members 


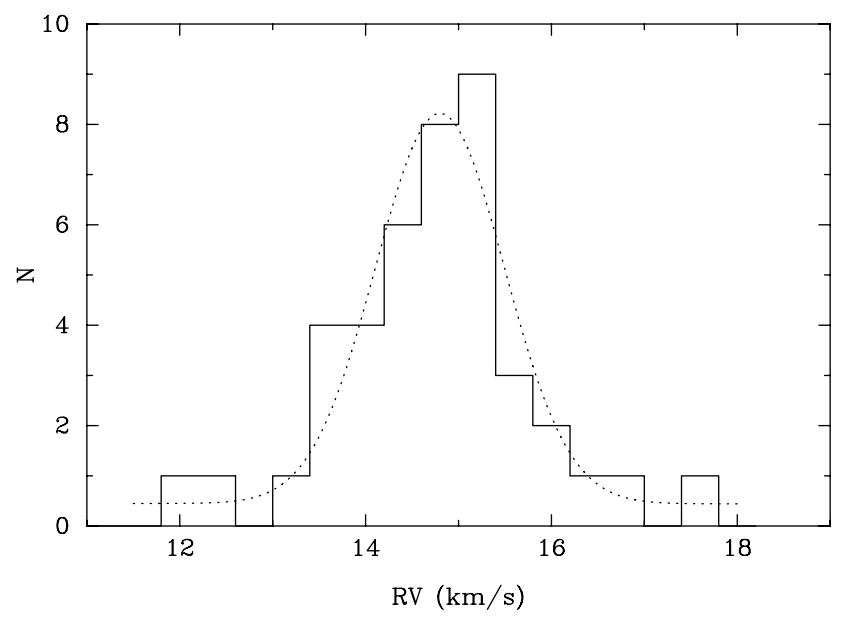

Fig. 4. Histogram of mean radial velocities for bona fide cluster members of IC 2391. The dashed line shows a Gaussian fit that peaks at $+14.80 \mathrm{~km} \mathrm{~s}^{-1}$.

that may have their instantaneous radial velocity outside the $3 \mathrm{~km} \mathrm{~s}^{-1}$ window. If a star is a known or suspected binary, this criterion is relaxed.

4. At the age of IC 2391 ( $\sim 40 \mathrm{Myr}$ ), the stars cooler than $T_{\text {eff }} \sim$ $7000 \mathrm{~K}$ must have a Li I feature in their spectra at $6708 \AA$. The lack of lithium indicates that a star is a much older field star. The Li test could not be applied to several fainter stars due to the lack of high-resolution spectra.

If any of the criteria listed above fails, such a star may not qualify for a list of bona fide cluster members. In the case of doublelined spectroscopic binaries their barycentric velocity should be within $\sim 5 \mathrm{~km} \mathrm{~s}^{-1}$ around the cluster's mean velocity. Thus, a total of 66 bona fide cluster members are selected and denoted by an asterisk in the last column of Table 1 . For convenience, we also provide cross-identifications with HD stars (numbers greater than 70000) in Table 1 and the VXR numbers from Patten \& Simon (1996). It is still possible that a few stars among these stars may not survive further scrutiny for cluster membership, and some rejected stars may turn out to be genuine cluster members.

A list of bona fide cluster members was used to find the cluster center, previously not very well known for IC 2391. We assumed a Gaussian profile to model marginal distributions in right ascension and declination of the observed star spatial density. A fit to these distributions yields a cluster center, equal to $\alpha=8^{\mathrm{h}} 41^{\mathrm{m}} 00^{\mathrm{s}} .3$ and $\delta=-53^{\circ} 00^{\prime} 36^{\prime \prime}(\mathrm{J} 2000.0)$. Due to a highly asymmetric distribution of cluster members, the uncertainty in the cluster center is about $1^{\prime}$.

The sample of bona fide cluster members allowed us to reexamine the distribution of radial velocities drawn nearly exclusively from this study only, i.e., the merged list of CORAVEL and FEROS radial velocities. We selected 42 stars showing no apparent signs of duplicity. The distribution of radial velocities was binned in $0.4 \mathrm{~km} \mathrm{~s}^{-1}$ increments and then fitted with a Gaussian (Fig. 4). The best fit yields a mean radial velocity of $14.80 \pm 0.69 \mathrm{~km} \mathrm{~s}^{-1}$ (s.d.). This is very close to the mean radial velocity of $14.6 \mathrm{~km} \mathrm{~s}^{-1}$ estimated from the Stauffer et al. (1997) data. The distribution of radial velocities appears to be slightly skewed. We believe that small number statistics is a primary source of this skewness, although stars with $v \sin i>90 \mathrm{~km}^{-1}$ may also contribute to this effect by having their radial velocity slightly increased.
A few stars in Tables 4 and 8 that have their radial velocities very close to the cluster's mean velocity fail one or two additional criteria. Two of them, 2717 and 5314, are located below the main sequence but otherwise would qualify for cluster membership and probably deserve further scrutiny. The third star, 5376, is almost a perfect cluster member, notwithstanding the noted absence of lithium. The available spectrum is rather noisy; therefore, we cannot be confident about the lack of Li feature, so additional checks are required. According to Randich et al. (2001), the cluster member $4636=$ SHJM 9, only by $\sim 100 \mathrm{~K}$ hotter than star 5376, has a fairly prominent Li 6708 feature with $E W(\mathrm{Li})=100 \mathrm{m \AA}$. Hence, there is no reason to assume that $\mathrm{Li}$ would be depleted below detection in star 5376, if it were a cluster member.

\subsection{Membership status of Patten \& Simon and SHJM stars}

Many of the recent advances in the understanding of IC 2391 rest on the X-ray source list from ROSAT observations (Patten \& Simon 1993, 1996). A total of 80 sources have been identified from these observations. Owing to a rather low spatial resolution ( 5-30" FWHM) for ROSAT detectors, identification of optical counterparts in many cases is uncertain. To ensure that none of the possible counterparts with $V<19$ is missing, Patten $\&$ Simon (1996) provide a total of 184 possible identifications and then try to narrow down the list using available proper motions, photometry, and spectroscopy. In essence, the papers by Stauffer et al. (1997), analyzing 26 counterparts, and Randich et al. (2001), re-analyzing a subsample from the Stauffer et al. paper, is a continuation of this effort. We have astrometric data for 49 optical counterparts, all brighter than $V \sim 15$ (see crossidentifications in Table 9). Among these stars, 12 are field stars with the membership probability, $P_{\mu}=0$. A formal sum of probabilities indicates that the expected number of cluster members is $\sim 25$. This estimate is lower than the actual number of members because we could not incorporate radial distribution in the membership calculation (see Sect. 2). Indeed, there are 34 bona fide cluster members among the likely optical counterparts of X-ray sources. We conclude that, while proven successful in finding many low-mass cluster members, the X-ray activity alone is not a decisive and comprehensive membership criterion.

To illustrate how deceptive corroborative evidence can sometimes be, consider star $6576=\mathrm{WXR} 50 \mathrm{a}$. It is listed as a "suspected cluster member based on photometry and/or spectroscopy" by Patten \& Simon (1996). Its radial velocity from our study is $+15.13 \mathrm{~km} \mathrm{~s}^{-1}$ and $+17 \mathrm{~km} \mathrm{~s}^{-1}$ (Stauffer et al. 1997), both very close to the cluster's mean radial velocity. For this star, the Li abundance is 2.7 (our study) and 2.6 according to Randich et al. (2001), tightly following the $\log N(\mathrm{Li})$ vs. $T_{\text {eff }}$ trend for IC 2391 (Fig. 3). A closer inspection of color-magnitude diagrams $V,(V-I)$ and $J,(J-K)$ shows that a star is located $\sim 0.5$ mag below the main sequence. However, it is the proper motion of $6576\left(\mu_{x}=-8.8\right.$ and $\mu_{y}=+2.6$ mas yr $\left.^{-1}\right)$ that, at $11 \sigma_{c}$ apart from the cluster's mean motion, rules out the cluster membership.

One star, $4658=$ VXR $45 \mathrm{a}$, warrants a special statement. While it is not listed by us as a bona fide member because it is located relatively far above the main sequence in the colormagnitude diagram, its high proper-motion membership probability of $P_{\mu}=75 \%$ strongly suggests the cluster membership. This G9 spectral type star, also a BY Dra type variable V370 Vel, stands out by its huge rotational velocity of $v \sin i=238 \mathrm{~km} \mathrm{~s}^{-1}$ (Marsden et al. 2004), by far exceeding any other $v \sin i$ 

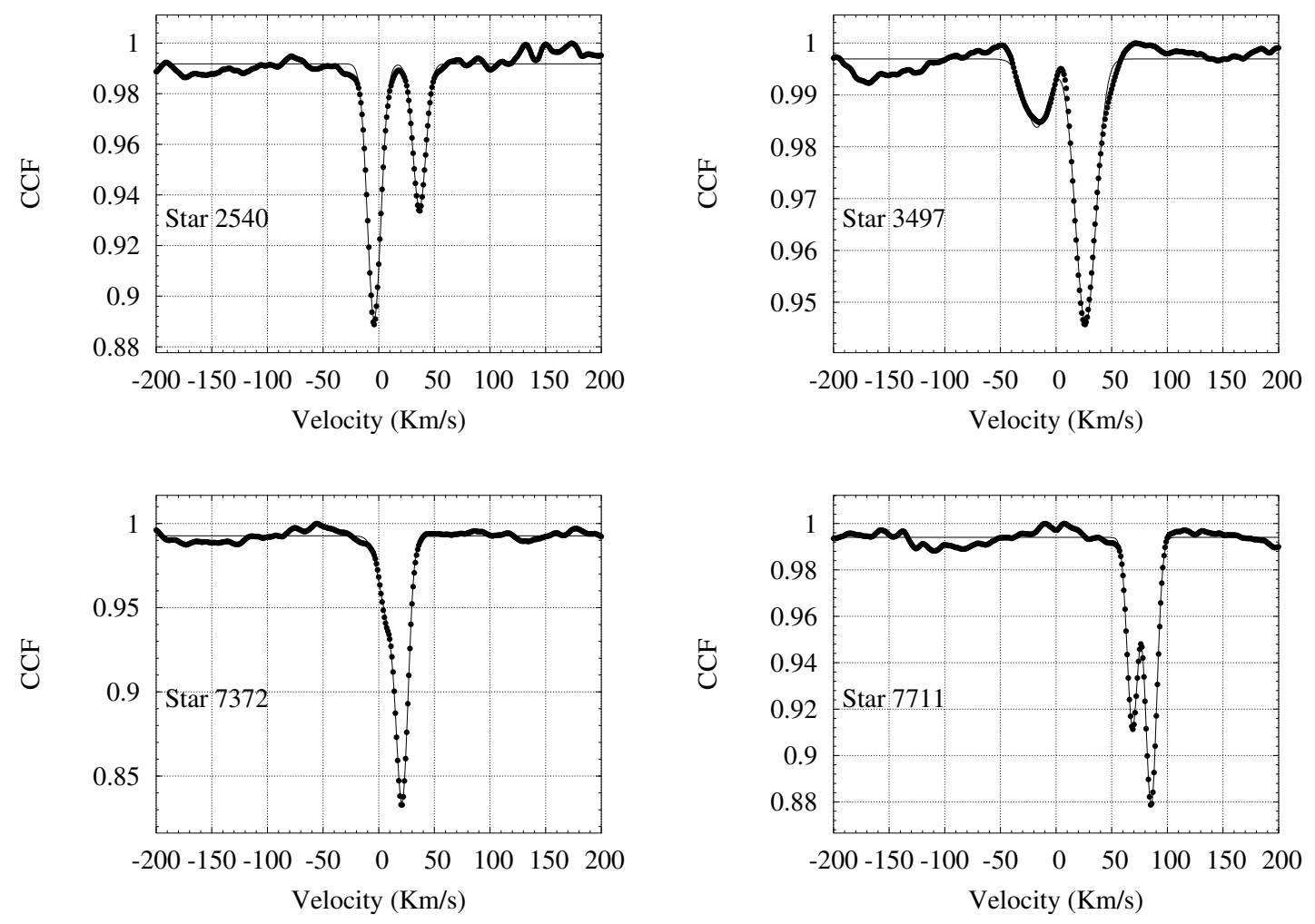

Fig. 5. Examples of cross-correlation function for double-lined spectroscopic binaries found among the FEROS target stars.

measurement in IC 2391 (Stauffer et al. 1997, see also Tables 4 and 8). The short photometric rotational period equal to $P_{\text {rot }}=$ 0.223 days (Patten \& Simon 1996) is also clearly visible in the $\mathrm{X}$-ray time series observations (Marino et al. 2003). If this is a genuine cluster member, then the question is what mechanism spun it up or prevented the dissipation of its primordial angular momentum, while most cluster members similar to star 4658 are slow rotators $\left(v \sin i<20 \mathrm{~km} \mathrm{~s}^{-1}\right)$. It should be noted that such ultrafast rotators can be found in other young open clusters, e.g., in the Pleiades (van Leeuwen et al. 1987), $\alpha$ Per (Prosser 1992), IC 2602 (Stauffer et al. 1997). One possible explanation of this phenomenon is offered by Barnes \& Sofia (1996), invoking a paradigm of the angular momentum loss saturation.

There is another fast rotator VXR 80a with $v \sin i \sim 150 \mathrm{~km} \mathrm{~s}^{-1}$ (Stauffer et al. 1997) among the suspected cluster members by Patten \& Simon (1996). Since we have not measured this star, the only source of proper motion for VXR $80 \mathrm{a}=$ King 391 is a study by King (1979). In this paper the cluster's proper motion centroid is at $\mu_{x}=-17.0$

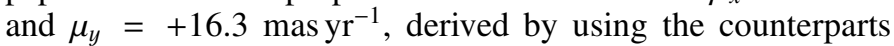
of our bona fide cluster members in King (1979). If we adopt the standard error of proper motions $\sigma=1.5 \mathrm{mas} \mathrm{yr}^{-1}$, then the proper motion of King 391 is more than $6 \sigma$ apart from the cluster's mean motion which rules out the cluster membership.

The star SHJM 5 also shows $v \sin i \sim 150 \mathrm{~km} \mathrm{~s}^{-1}$ (Stauffer et al. 1989). This star is a member of a visual binary (our star 4757) for which we have the membership probability $P_{\mu}=$ $28 \%$, consistent with cluster membership. Finally, star 2457 with $v \sin i=177 \mathrm{~km} \mathrm{~s}^{-1}$ is another possible cluster member despite its measured radial velocity at $-7.25 \mathrm{~km} \mathrm{~s}^{-1}$, since its astrometric membership probability is $P_{\mu}=71 \%$ and it is located just slightly above the main sequence in both $B V$ and $J K$ colormagnitude diagrams.
Prior to ROSAT measurements, the so-called SHJM list of 10 relatively faint cluster members was published by Stauffer et al. (1989). Cross-identifications of SHJM stars are provided by Patten \& Simon (1996). Based on our astrometry and criteria listed in Sect. 6.1, stars SHJM 1, 2, 6, 9, 10 appear to be bona fide cluster members, while SHJM 8 was not measured but SHJM $7=3664$ has a membership probability of zero. The membership for SHJM 3=1820 and (SHJM 4+SHJM 5) $=4757$ is not definitely constrained by our data. For SHJM 3 this is because its radial velocity from our data is $+12.76 \mathrm{~km} \mathrm{~s}^{-1}$, while Stauffer et al. (1989) provide $+19.5 \mathrm{~km} \mathrm{~s}^{-1}$, thus indicating a possible spectroscopic binary. Its astrometric membership probability is only $9 \%$, which is more a characteristic of a field star. Our conservative lower limit of equivalent width for the Li feature for SHJM 3 is 2.5 times larger than the measurement by Stauffer et al. (1989), thus implying unusually high abundance of $\mathrm{Li}$ at $T_{\text {eff }}=4480 \mathrm{~K}$ in the context of the overall $\log N(\mathrm{Li})$ vs. $T_{\text {eff }}$ curve for IC 2391 (see Fig. 3).

\subsection{Membership of some bright stars}

3518 = HIP 42504: a 5th magnitude star whose Hipparcos astrometry is consistent with cluster membership. Its redward position from the main sequence in the $B V$ and $J K$ CMDs (see Sect. 6.5) indicates duplicity, which is confirmed by radial velocity measurements (Feinstein 1961; Levato et al. 1988). The star is an SB1 spectroscopic binary with $\mathrm{P}=3.2 \mathrm{~d}$, eccentricity $e=0.05$, and $\gamma$-velocity $=14.5 \mathrm{~km} \mathrm{~s}^{-1}$.

3658 = HD 74438: this star is $\sim 0.9$ magnitudes above the main sequence in the color-magnitude diagram, indicating a potential triple system. Its proper-motion membership probability $P_{\mu}=88 \%$ is high and reliable. Feinstein (1961) lists two measurements of radial velocity: +7.5 and $+17.5 \mathrm{~km} \mathrm{~s}^{-1}$, whereas 
Buscombe (1965) derives $+21: \mathrm{km} \mathrm{s}^{-1}$ from five spectrograms. Thus, the scatter of radial velocities is indicative of a non-single status of the star. A formal mean velocity matches the cluster's radial velocity. We note that at the XMM-Newton observatory Marino et al. (2005) were able to obtain only the upper limit of X-ray luminosity for this star, which is typical of A spectral type stars. On the grounds of the available data, we consider star 3658 a bona fide cluster member, albeit one requiring more spectroscopic studies.

4484 = HIP 42536 $=o$ Velorum: the brightest star in IC 2391, also a $\beta$ CMa type variable. Its Hipparcos parallax $\pi=6.59 \pm 0.51$ mas is very close to the mean parallax of IC 2391 equal to $6.85 \pm 0.22$ (Robichon et al. 1999); however, Hipparcos

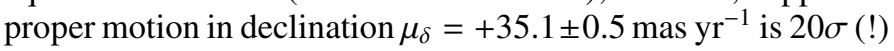
apart from the cluster's mean proper motion $\mu_{\delta}=+22.7 \pm 0.2$ (Robichon et al. 1999). Gontcharov et al. (2001) report a significantly lower absolute proper motion $\mu_{\delta}=+23.6 \pm 2.0$ consistent with Robichon et al. (1999). Our membership probability, $P_{\mu}=81 \%$, and the mean radial velocity of $+15.2 \mathrm{~km} \mathrm{~s}^{-1}$ by van Hoof (1972) together indicate that the star is a bona fide cluster member. The discrepant Hipparcos proper motion can be explained as the effect of unresolved binarity (Wielen et al. 1999).

5459= HD 74009: a star with lower membership probability $\left(P_{\mu}=25 \%\right)$ than the other relatively bright stars. However, the mean radial velocity of $+14 \mathrm{~km} \mathrm{~s}^{-1}$ by Levato et al. (1988) strongly supports cluster membership. Apparent variability of the observed radial velocity (Levato et al. 1988) indicates a possible spectroscopic binary.

7847 = HIP 42823: another bright star in IC 2391 first considered to be a member by Eggen (1991). Its Hipparcos parallax is only $0.7 \sigma$ apart from the mean parallax of IC 2391 , while Hipparcos proper motion deviates from the mean by $\sim 3$ mas $\mathrm{yr}^{-1}$ in both coordinates. Our membership probability of $78 \%$ supports the association of star 7847 with IC 2391, however, the lack of radial velocity measurements prevents us from assigning it the status of a definite cluster member.

\subsection{Spectroscopic binaries}

Identifying spectroscopic binaries in star clusters has a dual purpose. First, the evolutionary paths of stars are reasonably wellunderstood only for single stars; therefore, it is critical that we can identify these stars in the color-magnitude diagram with high confidence. Radial velocities from high-resolution spectroscopy is a powerful tool for detecting close binaries in a wide range of mass ratios. Second, the binaries themselves are very important to astronomy, especially those in the star clusters that provide a coeval sample of stars in a wide range of masses, all having the same initial composition.

The early spectroscopic work in IC 2391 was focussed on bright, early type stars (Feinstein 1961; Buscombe 1965; Perry \& Bond 1969; van Hoof 1972; Levato et al. 1988). In Table 1 - last column - we list only double-lined spectroscopic binaries (SB2), single-lined spectroscopic binaries (SB1 or SB as listed in the source), and suspected spectroscopic binaries (SB:), if a star shows a variable radial velocity or the mean radial velocity in different studies is significantly variant. The binaries with orbital solutions are marked with a letter "o". Since the internal mean error in these studies can reach up to $3-5 \mathrm{~km} \mathrm{~s}^{-1}$, not all cases of suspected SB may be real.

Among the IC 2391 F-K type main sequence stars, the long-term CORAVEL observations have revealed two SB2 and
Table 10. Orbital elements of $4413=$ VXR 5.

\begin{tabular}{lll}
\hline \hline Element (units) & Value & $\sigma$ \\
\hline$P(\mathrm{~d})$ & 90.617 & 0.007 \\
$T(\mathrm{JD}-2400000)$ & 45025.79 & 0.51 \\
$e$ & 0.287 & 0.007 \\
$\gamma$-velocity $\left(\mathrm{km} \mathrm{s}^{-1}\right)$ & 14.35 & 0.09 \\
$\omega\left({ }^{\circ}\right)$ & 22.8 & 1.3 \\
$K_{1}\left(\mathrm{~km} \mathrm{~s}^{-1}\right)$ & 29.51 & 0.25 \\
$K_{2}\left(\mathrm{~km} \mathrm{~s}^{-1}\right)$ & 30.54 & 0.26 \\
$a_{1} \sin i(\mathrm{Gm})$ & 35.23 & 0.38 \\
$a_{2} \sin i(\mathrm{Gm})$ & 36.45 & 0.39 \\
$\sigma(\mathrm{O}-\mathrm{C})\left(\mathrm{km} \mathrm{s}^{-1}\right)$ & 0.60 & $\cdots$ \\
$n_{\text {obs }}$ & 22 & $\cdots$ \\
\hline
\end{tabular}

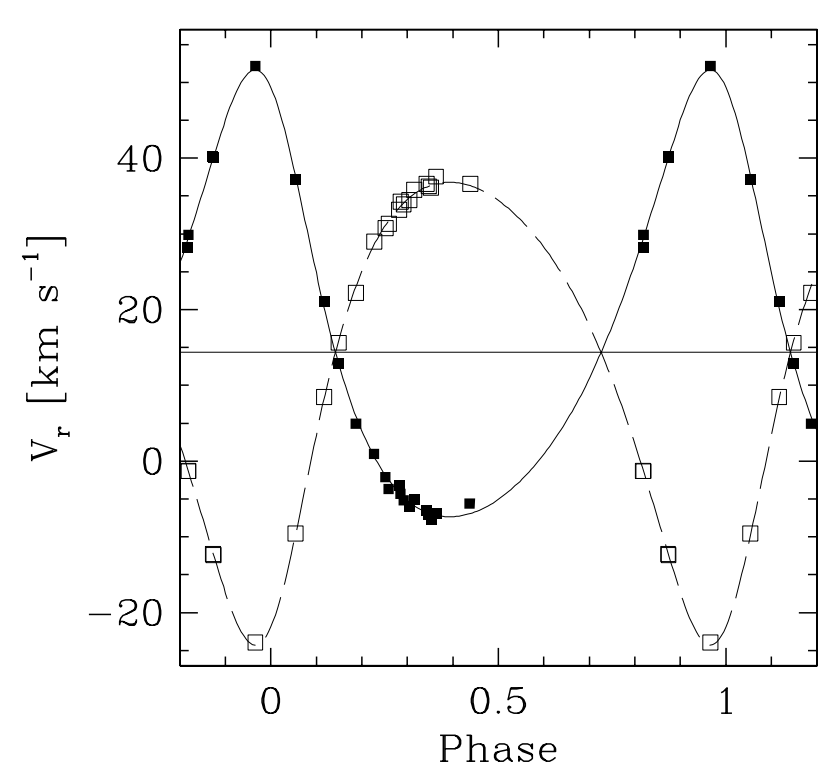

Fig. 6. Radial-velocity curve for spectroscopic binary star 4413 convolved with the orbital period of $\mathrm{P}=90.617$ days. The filled squares denote component A; open squares - component B.

four SB1 spectroscopic binaries (see Tables 3 and 4). An SB2 spectroscopic binary 4413 has a sufficient number of observations to calculate the orbital elements listed in Table 10. This binary system (Fig. 6) consists of two nearly equal-mass components on a fairly eccentric orbit $(e \sim 0.3)$. Its $\gamma$-velocity of $14.35 \mathrm{~km} \mathrm{~s}^{-1}$ clearly supports the cluster membership.

According to its proper motion, $\mu_{x}=-4.0$ and $\mu_{y}=$ $+11.8 \mathrm{mas} \mathrm{yr}^{-1}$, the SB2 spectroscopic binary 389 is a field star. As indicated by CORAVEL observations in Table 3, this binary was not resolved over four epochs, and these observations yield an estimate of the $\gamma$-velocity at $9.27 \mathrm{~km} \mathrm{~s}^{-1}$ (Table 4). The Wilson (1941) method allows us to determine the $\gamma$-velocity more precisely and to obtain the mass ratio in this binary system. Thus, the refined $\gamma$-velocity is $+10.24 \mathrm{~km} \mathrm{~s}^{-1}\left(\mathrm{~K}_{1}=19.36\right.$ and $\mathrm{K}_{2}=21.75 \mathrm{~km} \mathrm{~s}^{-1}$ ) and the mass ratio $M_{a} / M_{b}=0.89$. Clearly, the $\gamma$-velocity of star 389 also rules out cluster membership.

Finally, the FEROS high-resolution spectra obtained in one or two epochs (Table 5) indicate five SB2 binaries, four of them $(819,2540,3497,7372)$ are bona fide cluster members. For a set of four FEROS double-lined spectroscopic binaries, the observed cross-correlation functions are shown in Fig. 5. One of them (star 7711) has a barycentric velocity clearly incompatible with the mean radial velocity of the cluster.

Altogether, among 66 bona fide members, there are seven $\mathrm{SB} 2$, six SB1, and nine suspected spectroscopic binaries. If 


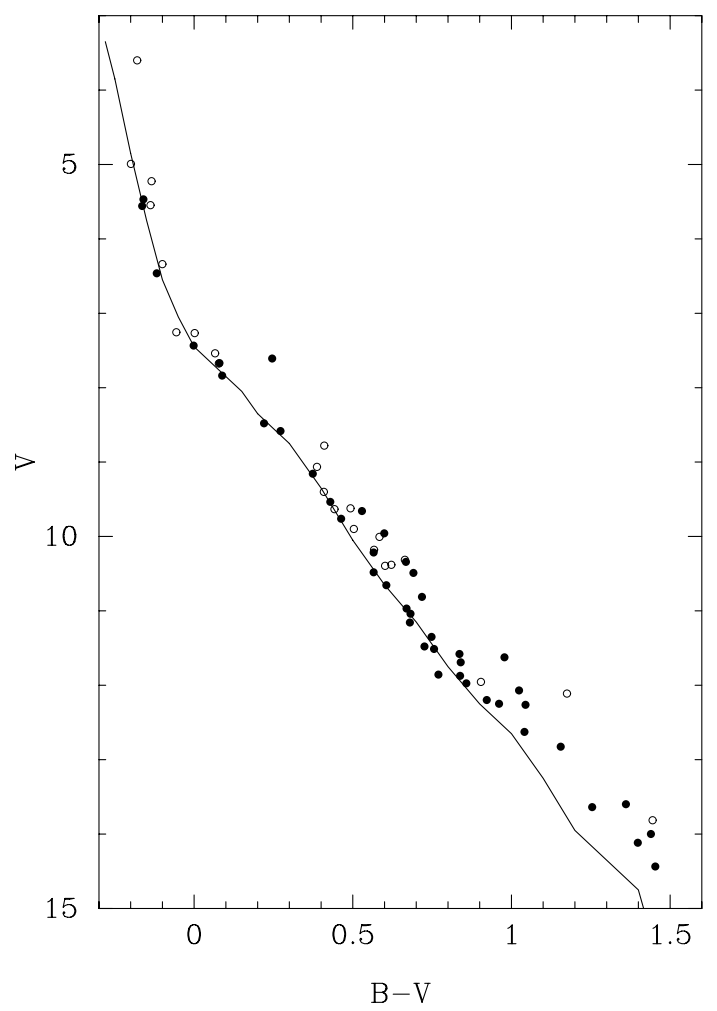

Fig. 7. Color-magnitude diagram for the bona fide members of IC 2391. The bold dots denote single stars or the stars with unknown multiplity status. The open circles indicate spectroscopic binaries. Zero-age main sequence is overplotted using the distance modulus equal to $5.95 \mathrm{mag}$ and zero reddening, $E(B-V)=0.0$.

all spectroscopic binary categories are considered, then the binary frequency in IC 2391 is at least $\sim 30 \%$. The location of all 22 spectroscopic binaries in $B V$ color-magnitude diagram is shown in Fig. 7.

\subsection{Color-magnitude diagram}

There are two sources of complete photometry for the bona fide cluster members: our CCD $B V$ and 2MASS $J H K_{\mathrm{s}}$ (Skrutskie et al. 2006) photometry. In the case of a missing CCD $B V$ photometry value, we used photoelectric $B V$ photometry from the literature. Such values in Table 1 are recognizeable by having only two decimal digits. The best previous CMD in $U B V$ is presented by Perry \& Hill (1969). These authors conclude that IC 2391 is unreddened, i.e, $E(B-V)=0.00$. It was later confirmed by measurements in the Vilnius seven-color photometric system (Forbes et al. 1998) and is also adopted in the present paper. The true reddening probably is not exactly zero; however, the limited precision of existing $U B V$ photometry and still unclear binarity status of some early spectral type cluster members preclude us from deriving a better reddening estimate. The following fits to the CMD, however, do not support the high value of $E(B-V)=0.06$ reported by Barrado y Navascués et al. (2004). The studies by Stauffer et al. $(1989,1997)$ and Patten \& Simon (1996) mainly employ VI photometry in constructing the CMD of IC 2391, assuming a distance modulus $(m-M)=6.05$. We note that the latter is 0.23 mag larger than the distance modulus from Hipparcos parallaxes (Robichon et al. 1999). The color-magnitude diagrams in these studies indicate that the stars at $V \geq 11$ are located above the main sequence, presumably still being on the pre-main-sequence tracks. Our list of bona fide cluster members allows us to explore the properties of the $B V$ CMD (Fig. 7) in more detail.

We made a trial fit of ZAMS (Aller et al. 1982) to the $B V$ $\mathrm{CMD}$, assuming zero reddening, $E(B-V)=0.0$, and a distance modulus of $V_{0}-M_{V}=5.95$. It appears that bright stars are essentially unevolved, with the exception of the brightest member, star $4484=o$ Velorum, whose color is suspect due to possible duplicity (see Sect. 6.3). The lower main sequence seems to end at $V \gtrsim 12$ giving rise to pre-main sequence stars at fainter magnitudes. An exact interpretation of the $B V$ color-magnitude diagram for young open cluster clusters is complicated by the fact that $\mathrm{K}$ dwarfs in the Pleiades are either subluminous and/or have abnormally blue $B-V$ color (Stauffer et al. 2003), hence falling below the ZAMS. These authors suggest that all young $\mathrm{K}$ dwarfs may show a similar anomaly, thereby limiting the ability to obtain an estimate of the so-called PMS isochrone age. We examine this effect in the following section.

\subsubsection{Main-sequence fitting, distance modulus, age}

One of the goals of this study is to obtain a reliable photometric distance from the main sequence fitting. The empirical ZAMS used in Fig. 7 is not adequate for at least two reasons. First, the ZAMS from Aller et al. (1982) is based on the Hyades distance modulus of $m-M=3.28 \mathrm{mag}$, while Hipparcos parallaxes for the Hyades members yield $3.33 \mathrm{mag}$. Second, at the time of compiling this ZAMS, the metallicities of open clusters were poorly known. The uncertainty in $[\mathrm{Fe} / \mathrm{H}]$ is directly related to the uncertainty of the amount of the metal-line blanketing effect on colors and magnitudes - a major source of systematic errors in photometric distances.

Recently, Pinsonneault et al. $(2003,2004)$ and An et al. (2006) derived new isochrones specifically targeted to measuring the distances to open clusters and other parameters from the main-sequence fitting. They used the Yale Rotating Evolution Code (YREC) to construct stellar evolution tracks for masses $0.2 \leq M_{\odot} \leq 8$ and metallicities $-0.3 \leq[\mathrm{Fe} / \mathrm{H}] \leq+0.2$. The tracks were interpolated to provide theoretical isochrones for stellar ages from $20 \mathrm{Myr}$ to $4 \mathrm{Gyr}$, with the pre-main sequence phase included where appropriate. Then, the authors used the empirical $T_{\text {eff-color transformations from Lejeune et al. (1998) }}$ and applied small additional corrections to isochrones in order to match the photometry for the Hyades, adopting its metallicity at $[\mathrm{Fe} / \mathrm{H}]=+0.13 \mathrm{dex}$.

We used these isochrones to derive a precise distance modulus and to probe the range of possible cluster ages. The key parameter for assuring reliability of main-sequence fitting is metallicity. Our new spectroscopic estimate of $[\mathrm{Fe} / \mathrm{H}]$ for IC 2391 has an accuracy similar to the metallicities used by An et al. (2006) in their analysis of four open clusters. In previous studies the isochrone age of IC 2391 is estimated to be $~ 30-35 \mathrm{Myr}$ (Stauffer et al. 1997; Mermilliod 1981), while the location of the lithium-depletion boundary indicates an age of $50 \pm 5 \mathrm{Myr}$ (Barrado y Navascués et al. 2004). We chose 30, 40, and $55 \mathrm{Myr}$ isochrones from An et al. (2006) and interpolated them to match the cluster's metallicity of $[\mathrm{Fe} / \mathrm{H}]=+0.06 \mathrm{dex}$. These isochrones were fitted to the $B V$ CMD (Fig. 8) by adjusting them to the lower envelope of main sequence in the color range of $0.2<B-$ $V<0.7$ and by assuming zero reddening, $E(B-V)=0.00 \mathrm{mag}$. The resulting distance modulus is $V_{0}-M_{V}=6.01 \mathrm{mag}$, which is larger by $0.19 \mathrm{mag}$ or $2.7 \sigma$ than the distance modulus inferred from the mean parallax via the Hipparcos combined abscissae solution by Robichon et al. (1999) and van Leeuwen (1999). Following the argumentation by Pinsonneault et al. (1998) and 


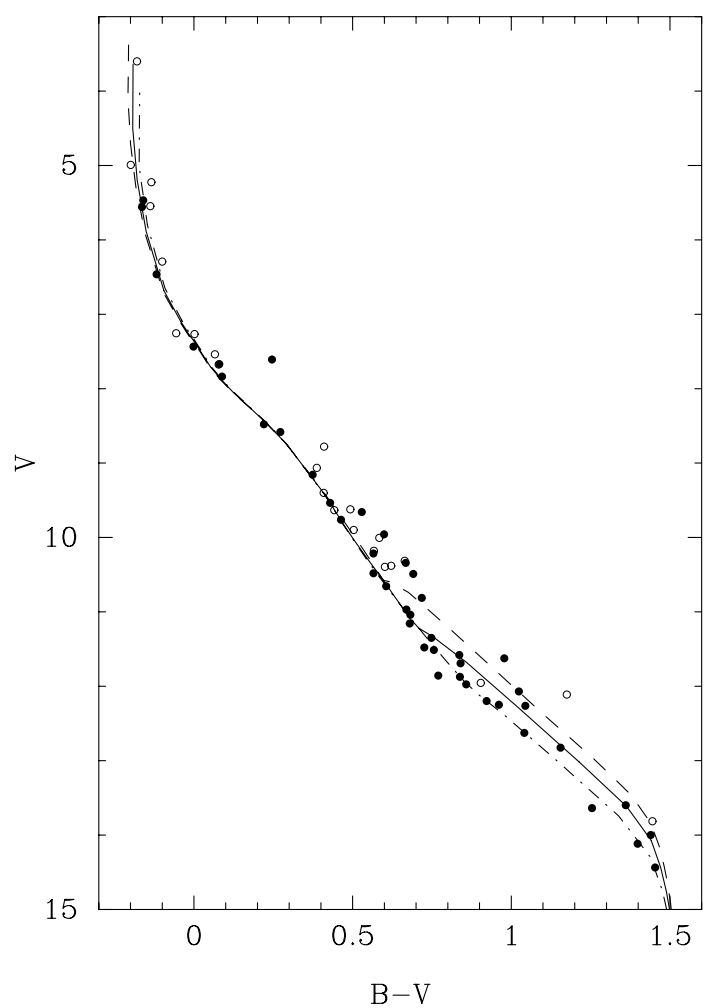

Fig. 8. Color-magnitude diagram for the bona fide members of IC 2391. See Fig. 7 for the meaning of bold dots and open circles. The fit of 30, 40 (dark curve), and $55 \mathrm{Myr}$ isochrones to CMD assuming zero reddening $(E(B-V)=0.0)$ yields the distance modulus of $V_{0}-M_{V}=6.01 \mathrm{mag}$. Our estimate of age is $40 \mathrm{Myr}$. The location of stars below the $40 \mathrm{Myr}$ isochrone at $V>11.5$ can be explained by anomalous spectral energy distributions in young $\mathrm{K}$ dwarfs.

the study of NGC 2451A (Platais et al. 2001), we adopted the uncertainty in our distance modulus to be $0.05 \mathrm{mag}$.

This result should be considered in the context of a trigonometric distance to the Pleiades from Hipparcos measurements vs. the Pleiades distance estimate from the main-sequence fitting. The Hipparcos measurements yielded a significantly smaller distance modulus for the Pleiades (i.e., $m-M=5.37 \pm$ 0.07 mag, equivalent to $118.3 \pm 3.5 \mathrm{pc}$ in van Leeuwen 1999) than the distance modulus from the main-sequence fitting, e.g., $m-M=5.60 \pm 0.04$ (Pinsonneault et al. 1998).

It is distressing to find another open cluster whose Hipparcos parallax distance is significantly shorter than the photometric distance. We point out that among the eleven IC 2391 members used by Robichon et al. (1999) to derive the mean cluster parallax, only one star (7027= HIP 42450) has a smaller Hipparcos parallax than our photometric parallax. Incidently, this star is the second faintest in the sample. What kind of systematic errors could possibly bias our photometric distance? The first is a small uncertainty in the reddening of IC 2391. If the true reddening is as high as $E(B-V)=0.01 \mathrm{mag}$, that alone would reduce the distance modulus by $0.03 \mathrm{mag}$. Second, if the true metallicity is solar, i.e., $[\mathrm{Fe} / \mathrm{H}]=0.0 \mathrm{dex}$, it would also reduce the distance modulus by an additional $\sim 0.08 \mathrm{mag}$. However, it is unlikely (although not imposssible) that either parameter is off by this much. Also, a major change in the stellar parameters would be required to lower the $[\mathrm{Fe} / \mathrm{H}]$ value enough to account for the $0.19 \mathrm{mag}$ discrepancy in the distance modulus.

In the light of another looming Hipparcos distance problem, it is instructive to have a look of how it is resolved for the Pleiades open cluster. The following diverse and independent studies actually all support the long distance to the Pleiades. Thus, Gatewood et al. (2000) measured trigonometric parallaxes of seven members of the Pleiades clusters and obtained an equivalent to the distance modulus $m-M=5.59 \pm 0.12$ mag. Using the Hubble Space Telescope's Fine Guidance Sensor, Soderblom et al. (2005) obtained absolute trigonometric parallaxes for three Pleiades members leading to the distance modulus $m-M=5.63 \pm 0.02$ mag. When Pan et al. (2004) applied Kepler's third law and the mass-luminosity relation to their interfermetric measurements, they found that the Pleiades visual binary star Atlas has $m-M=5.66 \pm 0.04 \mathrm{mag}$. A different set of interferometric observations for Atlas, combined with the measurements of radial velocities, produced better-constrained orbital elements and, consequently, a purely geometric distance or $m-M=5.60 \pm 0.07 \mathrm{mag}$ (Zwahlen et al. 2004). More strong evidence comes from the combined orbital solution of the eclipsing binary HD 23642, yielding the distance modulus $m-M=5.60 \pm 0.03 \mathrm{mag}$ (Munari et al. 2004). Finally, the frequency observations in six $\delta$ Scuti stars in the Pleiades match the eigenfrequencies of rotating stellar models best, when assuming $m-M=5.60-5.70$ (Fox Machado et al. 2006) and using the other generally adopted parameters of the Pleiades.

Regarding Hipparcos parallaxes, van Leeuwen (2005) indicates that correlations in the abscissa residuals for bright stars and weight disparities between each two fields-of-view in the attitude reconstruction process can be attributed to the problematic parallaxes for some open clusters, particularly the Pleiades. Makarov (2002) has shown that by accounting for the average residuals around the so-called reference circle helps to reduce the Pleiades Hipparcos parallax to an equivalent of $m-M=$ $5.55 \pm 0.06$. This, however, is not the final word since the raw Hipparcos data are under complete re-reduction (van Leeuwen \& Fantino 2005).

Our $B V$ color-magnitude diagram (Fig. 8) conclusively shows that the main sequence extends as deep as $V \sim 12$ or $M_{v}=+6$ and appears to populate the pre-main-sequence tracks at fainter magnitudes. If we adopt an age of IC 2391 equal to $40 \mathrm{Myr}$, our 40-Myr-old pre-main sequence isochrone (see Fig. 8) is located above the bulk of presumably premain-sequence stars. That could be explained by the observed "blueshift" of K dwarfs in the $B V$ CMD of Pleiades (Stauffer et al. 2003), noting that this effect in IC 2391 starts at $B-V \sim 0.7$ or at a spectral type G5. A 55-Myr old isochrone significantly mitigates this effect but, on the other hand, it does not fit the upper main sequence well, suggesting that the isochrone (nuclear) age is less than $55 \mathrm{Myr}$. In fact, the age estimate is constrained just by the single brightest cluster member $4484=o$ Velorum. Because of its suspected duplicity (see Sect. 6.3) and a $\beta \mathrm{CMa}$ type variability (van Hoof 1972), this star is not a typical B3 spectral type subgiant - its color $B-V=-0.18$ could be biased and, subsequently, affect the isochrone age. Summarizing, the age estimate from the $B V$ CMD could be anywhere between 30 and 50 Myr.

Recently Dodd (2004) attempted to use the 2MASS photometry to construct a CMD of IC 2391. One lesson learned from this exercise is that the accuracy of 2MASS photometry is inadequate at $H>14(J>15)$. Our bona fide cluster members are not fainter than $J=11.5$, so they can serve as building blocks in constructing an empirical ZAMS from the 2MASS data. The $J K$ color-magnitude diagram of IC 2391 (Fig. 9) has a considerably larger scatter across the main sequence than does the $B V \mathrm{CMD}$ (Fig. 8), possibly due to the higher sensitivity to a binary presence. It is quite possible that several undetected spectroscopic 


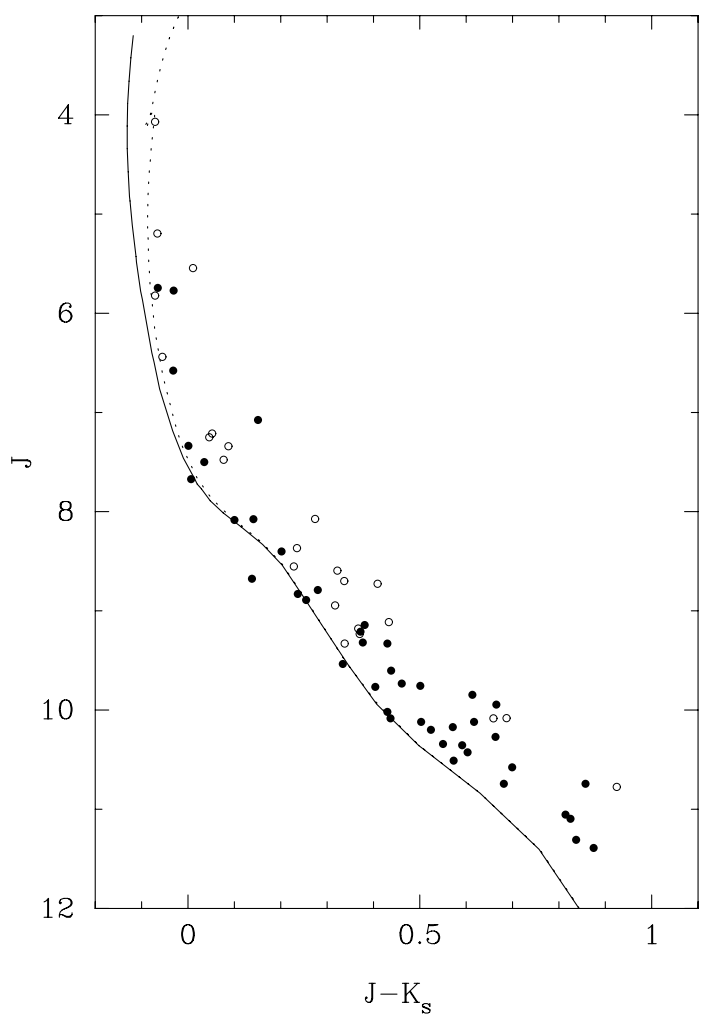

Fig. 9. Color-magnitude diagram for bona fide members of IC 2391 using 2MASS $J K$ photometry. See Fig. 7 for the meaning of bold dots and open circles. The reddening and distance modulus are the same as in Fig. 7. Two 40 and $100 \mathrm{Myr}$ solar metallicity isochrones from Bonatto et al. (2004) are overplotted.

binaries are still hidden in our sample of bona fide cluster members. Some other reasons for this scatter may include source confusion and different photometric measurement reductions for stars brighter and fainter than $J \sim 9$ (Cutri et al. 2003).

In the $J K$ CMD (Fig. 9), we overplotted 40 and $100 \mathrm{Myr}$ solar metallicity isochrones from Bonatto et al. (2004) using the same distance modulus as for the initial ZAMS fit in Fig. 7, i.e., $V_{0}-M_{V}=5.95$. Only a handful of stars are located right on the $40 \mathrm{Myr}$ isochrone, which itself poorly fits the upper main sequence. The best fit with a $100 \mathrm{Myr}$ isochrone is most likely compromised by some bias in the 2MASS photometry at bright magnitudes and/or by using a $T_{\text {eff-color transformation that is }}$ somewhat uncertain in this magnitude range. Thus, choosing a $100 \mathrm{Myr}$ isochrone helps to eliminate the mismatch at the bright end of main sequence; however, it is very unlikely that IC 2391 is indeed that old.

Following the referee's suggestion we investigated the $V K_{s}$ color-magnitude diagram. In these colors it is expected that the $\mathrm{K}$ dwarf "blueshift" in the CMD would disappear (Stauffer et al. 2003). Our VK CMD for bona fide cluster members is given in Fig. 10. We attempted to fit this CMD with 30,35,40,45,50,55Myr isochrones; however, a relatively poor match to the essential part of main sequence comprising F-G spectral type stars $\left(0.3 \leq V-K_{s} \leq 1.5\right)$ prevented us from improving the distance modulus over a similar fit of the $B V$ CMD. Hence, we adopted the distance modulus of $V_{0}-M_{V}=6.01$ obtained from the $B V$ CMD. It is difficult to judge what causes the scatter across the main sequence and the offset of its upper portion. Possibly, the 2MASS photometry contributes to these effects, but we also cannot entirely rule out small isochrone calibration problems for the $V-K_{s}$ color index. On the other hand, the lower part of color-magnitude diagram is indeed well-defined and allows us

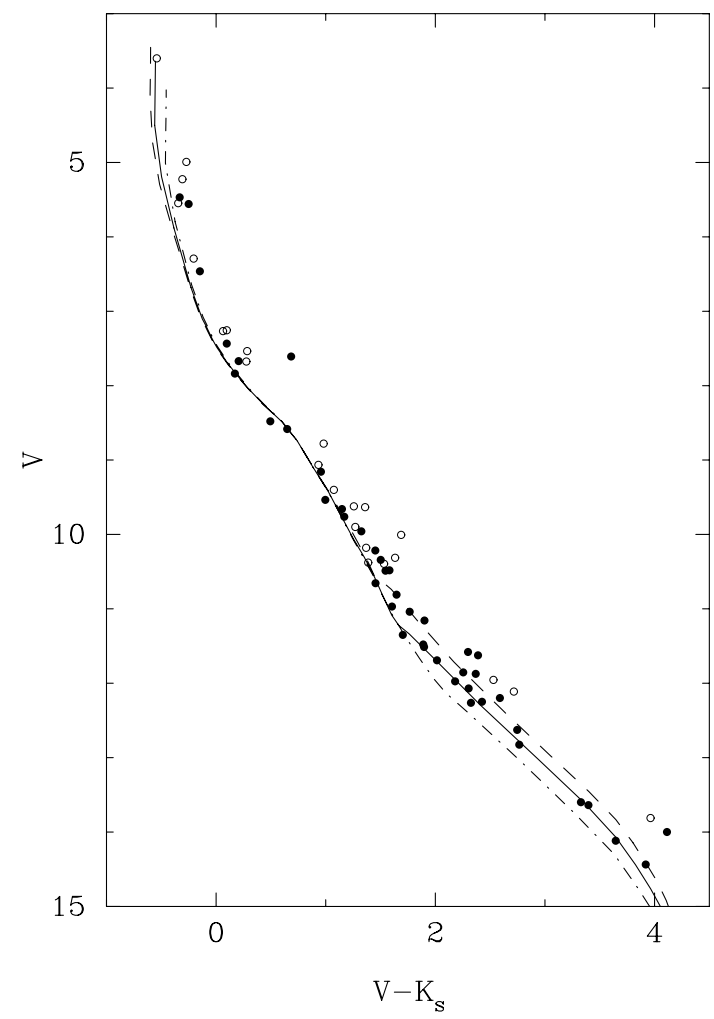

Fig. 10. $V K$ color-magnitude diagram for bona fide members of IC 2391. See Fig. 7 for the meaning of bold dots and open circles. The reddening and distance modulus are the same as for Fig. 8. The best fit to the lower part of CMD is provided by a $40 \mathrm{Myr}$ isochrone (dark curve). For comparison, 30 and $55 \mathrm{Myr}$ isochrones are also plotted. Similar to $J K C M D$, a fit to the upper part of main sequence is poor most likely for the same reasons as for the CMD from 2MASS photometry (see Fig. 9).

to confirm the best estimate of isochrone age for IC 2391 at $40 \mathrm{Myr}$.

The so-called lithium-depletion boundary (LDB) age method yields a higher age for IC 2391 at 50-53 Myr (Barrado y Navascués et al. 1999; Barrado y Navascués et al. 2004) and 50 Myr by Jeffries \& Oliveira (2005) using five different models. It should be noted that this is in line with similarly higher ages by this method for the $\alpha$ Per cluster (Stauffer et al. 1999) and the Pleiades (Stauffer et al. 1998). Since our data on IC 2391 do not reach the lithium-depletion boundary, we are not in a position to resolve the age differences.

\subsection{Rotational velocities $v \sin i$}

The distribution of $v \sin i$ for cluster members ranges from $\sim 2$ to $240 \mathrm{~km} \mathrm{~s}^{-1}$ (Fig. 11). Such a spread has already been observed by Stauffer et al. (1997) for the IC 2391+IC 2602 members and can be interpreted as a result of the early angular momentum evolution of low-mass stars, which appears to be regulated by the disk-locking mechanism (e.g., Edwards et al. 1993). According to this scenario, a gradual dissipation of the disk weakens the magnetic coupling between the star and its circumstellar disk, thereby releasing the star to spin up as it contracts during its premain-sequence phase.

Herbst \& Mundt (2005) analyzed a large data set of rotational periods for 500 low-mass stars in five nearby young open clusters (Orion nebula cluster, NGC 2264, $\alpha$ Per, IC 2602, and the Pleiades). They show that $50-60 \%$ of the stars still on the convective tracks, i.e., the vertical part of the pre-main-sequence tracks, appear to be released from the locking mechanism early 


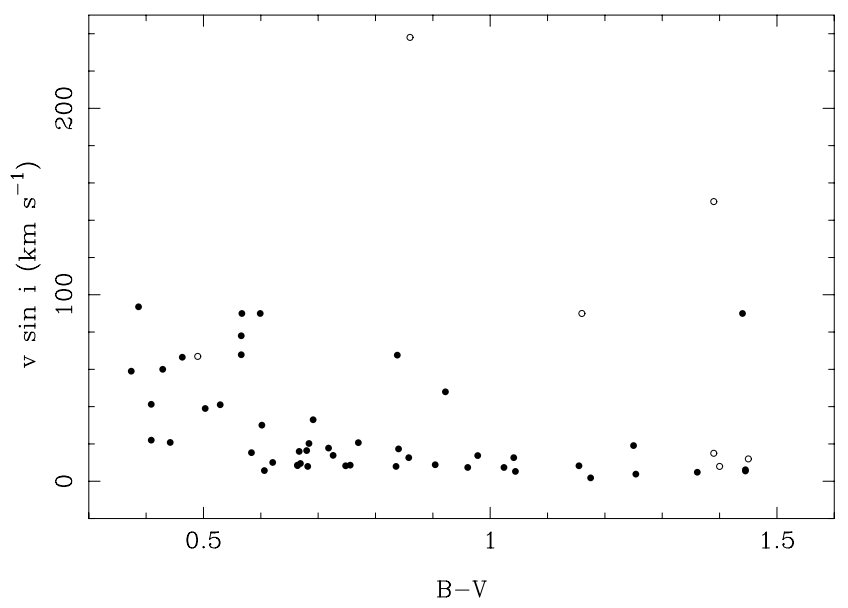

Fig. 11. Distribution of $v \sin i$ for bona fide cluster members as a function of $B-V$, which is roughly inversely proportional to luminosity. The filled circles are new measurements; open circles are those from the literature (fast rotators 1820, 4658, 4757 - likely but not bona fide cluster members - are also included). The distribution is bimodal, peaking at 10 and $\sim 80 \mathrm{~km} \mathrm{~s}^{-1}$. See Sect. 6.2 about the ultrafast rotator, star 4658 .

and thus account for the fast rotators. Conversely, the remaining stars lose a considerable amount of their angular momentum in the first few million years and enter onto the ZAMS as slow rotators. The distribution of $v \sin i$ in IC 2391 (Fig. 11) seemingly follows this pattern. However, a relatively short empirical life-time of circumstellar disks of $\sim 6 \mathrm{Myr}$ (Haisch et al. 2001) makes IC 2391, at its age of $40 \mathrm{Myr}$, an improper environment for testing the disk-locking mechanism. Recently, Barnes (2003) put forward a new interpretation of the observed pattern of rotational velocities (periods) in star clusters like IC 2391 and older. In this interpretive paradigm, the observed rotational morphology in $\mathrm{G}, \mathrm{K}$, and $\mathrm{M}$ stars along the so-called $I$ and $C$ sequences is driven by dynamos of two types that evolve in synchrony with the extent of the convective zone in stars. For IC 2391 the only source of rotational periods for 16 stars is the study by Patten $\&$ Simon (1996). We hope that the much larger sample of $v \sin i$ obtained in this study will help to advance the understanding of rotational evolution.

\section{Conclusions}

A decade ago Stauffer et al. (1997) commented that IC 2391, as well as IC 2602, are not giving up their secrets easily. Placed at an optimum age, these two clusters are ideal laboratories for studies of interface between the main sequence and late pre-main-sequence phases and their timescales. However, the intrinsic paucity of cluster members in both IC clusters is the main factor limiting the scientific return in the sense that even a single peculiar cluster member or an interloper may distort the observable trends and relationships. One such sensitive case is star 1820 (see Sect. 6.2) and its location on the Li abundance curve.

In this study we have significantly advanced the knowledge of IC 2391 in the range $-2<M_{v}<+8.5$ or down to $\sim 0.5 M_{\odot}$. This is a first extensive proper-motion study in the region of IC 2391 providing membership probabilities over a $9 \mathrm{deg}^{2}$ area, in effect assuring a high-degree of completeness in the chosen ranges of magnitudes, as explained in Sect. 2. We have measured the radial velocity for most of them. The new kinematic members mainly fill in the F2-K5 spectral region. The CORAVEL measurements of 31 stars considerably strengthen the membership status for most of them. Several spectroscopic binaries were detected; among them seven are double-lined SB2, with one yielding an orbital solution. The FEROS high-dispersion spectra served to measure radial velocities and projected rotational velocities and to estimate $\mathrm{Li}$ and $\mathrm{Fe}$ abundance. The latter is found to be $[\mathrm{Fe} / \mathrm{H}]=+0.06$ on the scale of solar abundance $\log \epsilon(\mathrm{Fe})=7.45 \mathrm{dex}$.

A total of 66 bona fide cluster members are selected by combining kinematic, spectroscopic, and photometric membership criteria. The $B V, J K$, and $V K$ color-magnitude diagrams were constructed using the bona fide cluster members. The main sequence fit yields a distance modulus of $V_{0}-M_{V}=6.01 \mathrm{mag}$ or $159.2 \mathrm{pc}$ that is significantly larger than the distance modulus and distance (5.82 mag and $146 \mathrm{pc}$ ) from the Hipparcos mean parallax for IC 2391.

The problem with Hipparcos mean cluster parallaxes could be quite complicated. For example, another nearby open cluster NGC $2451 \mathrm{~A}$, which is located only $18^{\circ}$ away from IC 2391 , does not show any discrepancy between the Hipparcos parallax and the distance modulus derived from the main sequence fitting (Platais et al. 2001), while the number of measured stars and their properties in both clusters are nearly identical. Both clusters are located near the ecliptic latitude of $\beta \sim-60^{\circ}$ and had been observed by Hipparcos relatively frequently and close in time. Comparative studies of these two open clusters may reveal clues to the cause of a $\sim 0.5$ mas offset in the Hipparcos mean parallax for IC 2391.

Among the most important tasks to advance the knowledge about IC 2391 are 1) extending reliable kinematic membership to fainter magnitudes and 2) accumulating high-resolution spectroscopy for all possible cluster members, especially for known or suspected spectroscopic binaries. The cluster population should be cleanly separated into single and binaries stars prior to extensive studies of individual stars and establishing a reliable distance to IC 2391 by other means.

Acknowledgements. We thank Hugo Levato for organizing the observing run at the CASLEO $2.2 \mathrm{~m}$ telescope. We appreciate helpful comments by Heiner Schwan and Peter De Cat about the properties of $o$ Velorum. We also thank Jeremy King and Simon Schuler for stimulating discussions on elemental abundances. We thank the referee, John Stauffer, for detailed and thoughtful comments that greatly helped to improve the interpretation of the results. We also thank Deokkeun An, Don Terndrup, and Marc Pinsonneault for their help with the new YREC isochrones generated at Ohio State University. This research made use of the SIMBAD database operated at the CDS, Strasbourg, France. This publication made use of data products from the Two Micron All Sky Survey, which is a joint project of the University of Massachusetts and the Infrared Processing and Analysis Center/California Institute of Technology, funded by the National Aeronautics and Space Administration and the National Science Foundation. I. Platais gratefully acknowledges support from the National Science Foundation through grant AST 04-06689 to Johns Hopkins University. J. Fulbright acknowledges support through grants from the W.M. Keck Foundation and the Gordon and Betty Moore Foundation, to establish a program of dataintensive science at to the Johns Hopkins University. M. Altmann and R. Mendez acknowledge the support by the Chilean Centro de Astrofísica FONDAP (15010003). The travel by J. Sperauskas to El Leoncito, Argentina was in part supported by NSF supplemental funding AST 01-39797. We heartily thank Lois J. Evans for meticulous editing of this paper.

\section{References}

Allende Prieto, C., Barklem, P. S., Lambert, D. L., \& Cunha, K. 2004, A\&A, 420,183

Aller, L. H., Appenzeller, I., Baschek, B., et al. 1982, Landolt-Börnstein: Numerical Data and Functional Relationships in Science and Technology New Series Group 6 Astronomy and Astrophysics, Vol. 2 (Landolt-Bornstein: Numerical Data and Functional Relationships in Science and Technology)

An, D., Terndrup, D. M., Pinsonneault, M. H., et al. 2006, ApJ, submitted [arXiv: astro-ph/0607549]

Baranne, A., Mayor, M., \& Poncet, J. L. 1979, Vistas Astron., 23, 279

Baranne, A., Queloz, D., Mayor, M., et al. 1996, A\&AS, 119, 373 
Barnes, S., \& Sofia, S. 1996, ApJ, 462, 746

Barnes, S. A. 2003, ApJ, 586, 464

Barrado y Navascués, D., Stauffer, J. R., \& Patten, B. M. 1999, ApJ, 522, L53

Barrado y Navascués, D., Stauffer, J. R., \& Jayawardhana, R. 2004, ApJ, 614, 386

Benz, W., \& Mayor, M. 1981, A\&A, 93, 235

Benz, W., \& Mayor, M. 1984, A\&A, 138, 183

Blair, M., \& Gilmore, G. 1982, PASP, 94, 742

Bonatto, C., Bica, E., \& Girardi, L. 2004, A\&A, 415, 571

Buscombe, W. 1965, MNRAS, 129, 411

Cutri, R. M., Skrutskie, M. F., van Dyk, S., et al. 2003, 2MASS All Sky Catalog of point sources. (The IRSA 2MASS All-Sky Point Source Catalog, NASA/IPAC Infrared Science Archive,

http://irsa.ipac.caltech.edu/applications/Gator/)

Dodd, R. J. 2004, MNRAS, 355, 959

Edwards, S., Strom, S. E., Hartigan, P., et al. 1993, AJ, 106, 372

Eggen, O. J. 1991, AJ, 102, 2028

Feinstein, A. 1961, PASP, 73, 410

Forbes, M. C., Dodd, R. J., \& Sullivan, D. J. 1998, Baltic Astronomy, 10, 375

Fox Machado, L., Pérez Hernández, F., Suárez, J. C., Michel, E., \& Lebreton, Y. 2006, A\&A, 446, 611

Fulbright, J. P., McWilliam, A., \& Rich, R. M. 2006, ApJ, 636, 821

Gatewood, G., de Jonge, J. K., \& Han, I. 2000, ApJ, 533, 938

Girard, T. M., Platais, I., Kozhurina-Platais, V., van Altena, W. F., \& López, C. E. 1998, AJ, 115, 855

Girardi, L., Bertelli, G., Bressan, A., et al. 2002, A\&A, 391, 195

Gontcharov, G. A., Andronova, A. A., Titov, O. A., \& Kornilov, E. V. 2001, A\&A, 365, 222

Gray, D. F. 1992, The observation and analysis of stellar photospheres (Cambridge Astrophysics Series, Cambridge: Cambridge University Press, 1992, 2nd ed., ISBN 0521403200.)

Haisch, K. E., Lada, E. A., \& Lada, C. J. 2001, ApJ, 553, L153

Herbst, W., \& Mundt, R. 2005, ApJ, 633, 967

Hogg, A. R. 1960, PASP, 72, 85

Jeffries, R. D., \& Oliveira, J. M. 2005, MNRAS, 358, 13

Kaufer, A., Stahl, O., Tubbesing, S., et al. 1999, The Messenger, 95, 8

King, D. S. 1979, Journal and Proceedings of the Royal Society of New South Wales, 112, 105

Kozhurina-Platais, V., Girard, T. M., Platais, I., et al. 1995, AJ, 109, 672

Kurucz, R. L., Furenlid, I., \& Brault, J. T. L. 1984, Solar flux atlas from 296 to $1300 \mathrm{~nm}$ (National Solar Observatory Atlas, Sunspot, New Mexico: National Solar Observatory, 1984)

Landolt, A. U. 1992, AJ, 104, 340

Lee, J.-F., \& van Altena, W. 1983, AJ, 88, 1683

Lejeune, T., Cuisinier, F., \& Buser, R. 1998, A\&AS, 130, 65

Levato, H., Garcia, B., Lousto, C., \& Morrell, N. 1988, Ap\&SS, 146, 361

Lyngå, G. 1960, Arkiv for Astronomi, 2, 379

Makarov, V. V. 2002, AJ, 124, 3299

Marino, A., Micela, G., Peres, G., Pillitteri, I., \& Sciortino, S. 2005, A\&A, 430, 287

Marino, A., Micela, G., Peres, G., \& Sciortino, S. 2003, A\&A, 407, L63

Marsden, S. C., Waite, I. A., Carter, B. D., \& Donati, J.-F. 2004, Astron. Nachr. 325,246

Martin, E. L. 1997, A\&A, 321, 492

Mathieu, R. D. 2000, in Stellar Clusters and Associations: Convection, Rotation, and Dynamos, ed. R. Pallavicini, G. Micela, \& S. Sciortino, ASP Conf. Ser., 198,517

Mayor, M. 1985, in Stellar Radial Velocities, ed. A. G. D. Philip, \& D. W. Latham, IAU Coll., 88, 35
Melo, C. H. F. 2003, A\&A, 410, 269

Melo, C. H. F., Pasquini, L., \& De Medeiros, J. R. 2001, A\&A, 375, 851

Mermilliod, J. C. 1981, A\&A, 97, 235

Mermilliod, J.-C., \& Mayor, M. 1989, A\&A, 219, 125

Munari, U., Dallaporta, S., Siviero, A., et al. 2004, A\&A, 418, L31

Nordstroem, B., Andersen, J., \& Andersen, M. I. 1996, A\&AS, 118, 407

Pan, X., Shao, M., \& Kulkarni, S. R. 2004, Nature, 427, 326

Patten, B. M., \& Simon, T. 1993, ApJ, 415, L123

Patten, B. M., \& Simon, T. 1996, ApJS, 106, 489

Paulson, D. B., Cochran, W. D., \& Hatzes, A. P. 2004, AJ, 127, 3579

Paulson, D. B., Sneden, C., \& Cochran, W. D. 2003, AJ, 125, 3185

Perry, C. L., \& Bond, H. E. 1969, PASP, 81, 629

Perry, C. L., \& Hill, G. 1969, AJ, 74, 899

Pinsonneault, M. H., Stauffer, J., Soderblom, D. R., King, J. R., \& Hanson, R. B. 1998, ApJ, 504, 170

Pinsonneault, M. H., Terndrup, D. M., Hanson, R. B., \& Stauffer, J. R. 2003, ApJ, 598, 588

Pinsonneault, M. H., Terndrup, D. M., Hanson, R. B., \& Stauffer, J. R. 2004, ApJ, 600, 946

Platais, I., Girard, T. M., Kozhurina-Platais, V., et al. 1998, AJ, 116, 2556

Platais, I., Kozhurina-Platais, V., Barnes, S., et al. 2001, AJ, 122, 1486

Prosser, C. F. 1992, AJ, 103, 488

Queloz, D. 1995, Ph.D. Thesis, Université de Genève

Ramírez, I., \& Meléndez, J. 2005, ApJ, 626, 465

Randich, S., Pallavicini, R., Meola, G., Stauffer, J. R., \& Balachandran, S. C. 2001, A\&A, 372, 862

Robichon, N., Arenou, F., Mermilliod, J.-C., \& Turon, C. 1999, A\&A, 345, 471

Schuler, S. C., King, J. R., Terndrup, D. M., et al. 2006, ApJ, 636, 432

Setiawan, J., Pasquini, L., da Silva, L., et al. 2000, The Messenger, 102, 13

Simon, T., \& Patten, B. M. 1998, PASP, 110, 283

Skrutskie, M. F., Cutri, R. M., Stiening, R., et al. 2006, AJ, 131, 1163

Sneden, C. A. 1973, Ph.D. Thesis

Soderblom, D. R., Jones, B. F., Balachandran, S., et al. 1993, AJ, 106, 1059

Soderblom, D. R., Nelan, E., Benedict, G. F., et al. 2005, AJ, 129, 1616

Stauffer, J., Hartmann, L. W., Jones, B. F., \& McNamara, B. R. 1989, ApJ, 342, 285

Stauffer, J. R., Barrado y Navascués, D., Bouvier, J., et al. 1999, ApJ, 527, 219

Stauffer, J. R., Hartmann, L. W., Prosser, C. F., et al. 1997, ApJ, 479, 776

Stauffer, J. R., Jones, B. F., Backman, D., et al. 2003, AJ, 126, 833

Stauffer, J. R., Schultz, G., \& Kirkpatrick, J. D. 1998, ApJ, 499, L199

Stütz, C., Bagnulo, S., Jehin, E., et al. 2006, A\&A, 451, 285

Udry, S., Mayor, M., \& Queloz, D. 1999, in IAU Coll. 170, Precise Stellar Radial Velocities, ed. J. B. Hearnshaw, \& C. D. Scarfe, ASP Conf. Ser., 185, 367

Upgren, A. R., Sperauskas, J., \& Boyle, R. P. 2002, Baltic Astronomy, 11, 91

van Hoof, A. 1972, A\&A, 18, 51

van Leeuwen, F. 1999, A\&A, 341, L71

van Leeuwen, F. 2005, in Transits of Venus: New Views of the Solar System and Galaxy, ed. D. W. Kurtz, IAU Coll., 196, 347

van Leeuwen, F., Alphenaar, P., \& Meys, J. J. M. 1987, A\&AS, 67, 483

van Leeuwen, F., \& Fantino, E. 2005, A\&A, 439, 791

Wielen, R., Dettbarn, C., Jahreiß, H., Lenhardt, H., \& Schwan, H. 1999, A\&A, 346,675

Wilson, O. C. 1941, ApJ, 93, 29

Yentis, D. J., Cruddace, R. G., Gursky, H., et al. 1992, in Digitised Optical Sky Surveys, ed. H. T. MacGillivray, \& E. B. Thomson, ASSL, 174, 67

Yong, D., Lambert, D. L., Allende Prieto, C., \& Paulson, D. B. 2004, ApJ, 603, 697

Zacharias, N., Urban, S. E., Zacharias, M. I., et al. 2004, AJ, 127, 3043

Zwahlen, N., North, P., Debernardi, Y., et al. 2004, A\&A, 425, L45 
I. Platais et al.: IC 2391, Online Material p 1

\section{Online Material}


Table 4. Mean radial velocities and vsini from Coravel observations.

\begin{tabular}{|c|c|c|c|c|c|c|c|c|c|c|}
\hline No & $\langle\mathrm{RV}\rangle$ & $\sigma$ & $\epsilon$ & $E / I$ & $n$ & $\Delta T$ & $v \sin i$ & $\sigma_{v \sin i}$ & $P\left(\chi^{2}\right)$ & Remarks \\
\hline 389 & 9.27 & 2.75 & 1.38 & 4.69 & 4 & 4412 & 14.4 & 1.8 & 0.000 & SB \\
\hline$\ldots$ & 5.00 & 8.35 & 2.52 & 10.49 & 11 & 3250 & 8.0 & 3.4 & 0.000 & SB2 (A) \\
\hline$\ldots$ & 17.00 & 10.83 & 3.27 & 7.39 & 11 & 3250 & 3.0 & 3.7 & 0.000 & SB2 (B) \\
\hline 736 & -25.11 & 3.17 & 3.17 & 1.00 & 1 & 0 & 22.1 & 11.2 & 9.999 & SB2 (A) \\
\hline$\ldots$ & 21.53 & 3.23 & 3.23 & 1.00 & 1 & 0 & 41.2 & 15.0 & 9.999 & SB2 (B) \\
\hline 1083 & 12.17 & 0.57 & 1.04 & 0.38 & 2 & 323 & 67.9 & 6.8 & 0.702 & \\
\hline 1142 & 13.99 & 0.42 & 0.42 & 1.00 & 1 & 0 & 7.9 & 1.7 & 9.999 & \\
\hline 1759 & 13.51 & 0.51 & 0.51 & 1.00 & 1 & 0 & 3.8 & 3.5 & 9.999 & \\
\hline 2012 & 13.90 & 0.63 & 0.63 & 1.00 & 1 & 0 & 17.4 & 1.6 & 9.999 & \\
\hline 2888 & 14.81 & 2.68 & 1.55 & 1.00 & 3 & 740 & 66.5 & 6.7 & 0.382 & \\
\hline 3464 & 13.93 & 2.77 & 1.05 & 2.78 & 7 & 4412 & 20.8 & 2.1 & 0.000 & SB \\
\hline 3497 & 31.69 & 0.65 & 0.65 & 1.00 & 1 & 0 & 11.8 & 2.5 & 9.999 & \\
\hline 3567 & 13.33 & 0.37 & 0.37 & 1.00 & 1 & 0 & 3.9 & 2.5 & 9.999 & \\
\hline 3623 & 24.05 & 0.37 & 0.37 & 1.00 & 1 & 0 & 4.2 & 2.3 & 9.999 & \\
\hline 3649 & 15.85 & 0.88 & 0.88 & 1.00 & 1 & 0 & 19.2 & 3.1 & 9.999 & \\
\hline 3664 & 6.70 & 2.28 & 1.14 & 4.42 & 4 & 2133 & 3.8 & 1.9 & 0.000 & SB \\
\hline 3722 & 15.25 & 4.74 & 1.43 & 9.33 & 11 & 4406 & 10.1 & 0.6 & 0.000 & SB \\
\hline 4336 & 15.63 & 0.41 & 0.41 & 1.00 & 1 & 0 & 8.0 & 1.9 & 9.999 & \\
\hline 4362 & 15.20 & 0.51 & 0.17 & 1.04 & 9 & 4412 & 9.0 & 0.7 & 0.401 & \\
\hline 4413 & 3.32 & 16.36 & 3.57 & 22.53 & 21 & 4412 & 8.6 & 0.7 & 0.000 & SB2 (A) \\
\hline$\ldots$ & 25.92 & 16.74 & 3.65 & 21.24 & 21 & 4412 & 8.4 & 0.7 & 0.000 & SB2 (B) \\
\hline 4467 & 15.22 & 0.19 & 0.32 & 0.33 & 3 & 2225 & 12.6 & 1.0 & 0.894 & \\
\hline 4549 & 16.59 & 17.81 & 6.73 & 8.31 & 7 & 4413 & 48.8 & 6.2 & 0.000 & SB \\
\hline 4636 & 13.63 & 0.52 & 0.52 & 1.00 & 1 & 0 & 4.9 & 2.9 & 9.999 & \\
\hline 4809 & 13.70 & 0.17 & 0.45 & 0.27 & 2 & 419 & 17.4 & 1.1 & 0.790 & \\
\hline 4902 & 14.71 & 0.59 & 0.42 & 1.01 & 2 & 418 & 8.3 & 1.8 & 0.313 & \\
\hline 5382 & 5.69 & 3.34 & 1.06 & 7.69 & 10 & 4411 & 8.7 & 0.8 & 0.000 & SB \\
\hline 5768 & 15.21 & 3.90 & 2.76 & 3.76 & 2 & 5 & 27.3 & 2.7 & 0.000 & SB \\
\hline 5829 & 10.03 & 1.65 & 1.65 & 1.00 & 1 & 0 & 67.6 & 9.3 & 9.999 & \\
\hline 5859 & 17.46 & 2.49 & 1.76 & 5.50 & 2 & 414 & 8.5 & 2.3 & 0.000 & SB \\
\hline 5884 & 14.49 & 0.56 & 0.40 & 1.01 & 2 & 419 & 13.9 & 1.1 & 0.313 & \\
\hline 6478 & 21.98 & 1.41 & 1.00 & 2.09 & 2 & 322 & 20.4 & 2.0 & 0.038 & \\
\hline 7794 & 9.38 & 0.57 & 0.37 & 0.58 & 7 & 4412 & 21.3 & 2.1 & 0.921 & \\
\hline 8415 & 15.73 & 0.66 & 0.66 & 1.00 & 1 & 0 & 20.7 & 2.3 & 9.999 & \\
\hline
\end{tabular}

Table 8. Mean data from FEROS spectroscopy.

\begin{tabular}{|c|c|c|c|c|c|c|c|c|c|c|c|c|c|}
\hline$\overline{\text { No }}$ & $\begin{array}{c}\langle R V\rangle \\
\left(\mathrm{km} \mathrm{s}^{-1}\right)\end{array}$ & $\begin{array}{c}v \sin i \\
\left(\mathrm{~km} \mathrm{~s}^{-1}\right)\end{array}$ & $\begin{array}{l}T_{\text {eff }} \\
\left({ }^{\circ} \mathrm{K}\right)\end{array}$ & $\begin{array}{c}E W(\mathrm{Li}) \\
(\mathrm{m} \AA)\end{array}$ & $\log N(\mathrm{Li})$ & $\begin{array}{c}E W\left(\mathrm{H}_{\alpha}\right) \\
(\AA)\end{array}$ & $\overline{\text { No }}$ & $\begin{array}{c}\langle R V\rangle \\
\left(\mathrm{km} \mathrm{s}^{-1}\right)\end{array}$ & $\begin{array}{c}v \sin i \\
\left(\mathrm{~km} \mathrm{~s}^{-1}\right)\end{array}$ & $\begin{array}{l}T_{\text {eff }} \\
\left({ }^{\circ} \mathrm{K}\right)\end{array}$ & $\begin{array}{c}E W(\mathrm{Li}) \\
(\mathrm{m} \AA)\end{array}$ & $\log N(\mathrm{Li})$ & $\begin{array}{c}E W\left(\mathrm{H}_{\alpha}\right) \\
(\AA)\end{array}$ \\
\hline 351 & 15.40 & 90.0 & 6024 & 90 & 2.82 & 3.69 & 4549 & 20.21 & 39.0 & 6192 & 150 & 3.36 & 2.9 \\
\hline 665 & 14.44 & 8.3 & 5451 & 189 & 2.88 & 2.11 & 4809 & 13.80 & 17.7 & 5546 & 175 & 2.90 & 3.1 \\
\hline 686 & 15.18 & 12.7 & 4538 & 215 & 1.87 & -0.13 & 5137 & 25.38 & 3.4 & 5148 & 0 & $\cdots$ & 1.5 \\
\hline 736 & 13.54 & 65.0 & 6579 & 83 & 3.22 & 5.10 & 5314 & 13.36 & 63.0 & 6467 & -99 & $\ldots$ & 5.62 \\
\hline 756 & 15.86 & 16.5 & 5357 & 217 & 2.96 & 2.7 & 5376 & 15.62 & 9.8 & 3936 & 0 & $\ldots$ & -1.17 \\
\hline 794 & 16.41 & 2.6 & 4874 & 0 & $\ldots$ & 1.4 & 5540 & 54.47 & 6.5 & 5652 & 48 & 2.13 & 3.10 \\
\hline 819 & 17.84 & 30.0 & 5764 & 179 & 3.15 & 3.7 & 5811 & 14.30 & 59.0 & 6823 & 107 & 3.58 & 4.84 \\
\hline 1174 & 13.54 & 60.0 & 6584 & 79 & 3.20 & 3.48 & 5859 & 16.86 & 8.8 & 4749 & 271 & 2.54 & -0.07 \\
\hline 1373 & 14.62 & 7.4 & 4746 & 156 & 1.83 & 1.0 & 6229 & 11.73 & 3.7 & 5200 & 61 & 1.78 & 2.1 \\
\hline 1560 & 15.98 & 5.7 & 5830 & 107 & 2.77 & 2.99 & 6576 & 15.13 & 56.0 & 5140 & 207 & 2.62 & 0.0 \\
\hline 1820 & 12.76 & 86.0 & 4479 & 333 & 2.58 & -0.6 & 6808 & 22.54 & 97.0 & 6652 & -99 & & 5.91 \\
\hline 2182 & 14.66 & 78.0 & 5920 & 187 & 3.35 & 3.0 & 6811 & 17.41 & 90.0 & 5952 & 137 & 3.07 & 3.65 \\
\hline 2456 & 14.82 & 48.0 & 4809 & 301 & 2.85 & -0.15 & 6974 & 15.38 & 5.3 & 4625 & 74 & 1.16 & 0.9 \\
\hline 2457 & -7.35 & 177.0 & 4527 & -99 & $\ldots$ & 0.0 & 6978 & 15.05 & 7.4 & 4668 & 179 & 1.84 & 0.17 \\
\hline 2540 & 15.14 & 6.2 & 3576 & 0 & $\ldots$ & -1.8 & 7372 & 15.80 & 1.8 & 4461 & 39 & 0.60 & 0.53 \\
\hline 2550 & -1.75 & 2.6 & 5053 & 0 & $\ldots$ & 1.6 & 7422 & 14.83 & 33.0 & 5651 & 233 & 3.40 & 2.47 \\
\hline 2578 & 27.78 & 2.9 & 4694 & 0 & $\ldots$ & 0.7 & 7442 & -19.82 & 2.3 & 4591 & 0 & $\ldots$ & 0.8 \\
\hline 2717 & 14.96 & 3.4 & 5267 & 24 & 1.41 & 3.4 & 7451 & 18.22 & 1.0 & 4519 & 0 & $\ldots$ & 1.60 \\
\hline 3359 & 14.69 & 8.6 & 5280 & 226 & 2.93 & 1.48 & 7663 & 25.70 & 5.4 & 5475 & 49 & 1.96 & 2.8 \\
\hline 3497 & -5.24 & 14.4 & 5622 & 126 & 2.68 & 2.66 & 7670 & 41.08 & 4.1 & 5281 & 63 & 1.89 & 2.3 \\
\hline 3695 & 15.16 & 90.0 & 3287 & -99 & $\ldots$ & -4.6 & 7711 & 73.58 & 4.0 & 5097 & 0 & $\ldots$ & 1.77 \\
\hline 4280 & 12.26 & 16.0 & 5729 & 151 & 2.94 & 3.05 & 7857 & 17.95 & 7.7 & 6072 & 114 & 3.03 & 2.82 \\
\hline 4324 & 14.31 & 41.0 & 6334 & 97 & 3.14 & 4.27 & 7956 & 15.29 & 13.8 & 4759 & 289 & 2.69 & -0.38 \\
\hline 4362 & 15.11 & 9.5 & 5616 & 191 & 3.08 & 2.39 & 7973 & 18.81 & 2.0 & 4730 & 10 & 0.35 & 1.71 \\
\hline 4413 & 14.48 & 11.4 & 5615 & 163 & 2.90 & 2.34 & 7974 & 19.80 & 7.5 & 5985 & 62 & 2.57 & 2.98 \\
\hline 4454 & 16.54 & 93.5 & 6761 & 83 & 3.35 & 6.61 & & & & & & & \\
\hline
\end{tabular}


Table 9. Cross-identifications with Patten \& Simon (1996).

\begin{tabular}{lcr|lcr}
\hline \hline VXR & No. & $P_{\mu}$ & VXR & No. & $P_{\mu}$ \\
\hline $2 \mathrm{~b}$ & 4336 & 73 & $45 \mathrm{a}$ & 4658 & 75 \\
$3 \mathrm{a}$ & 4362 & 67 & 46 & 3696 & 94 \\
4 & 3445 & 81 & 47 & 3695 & 80 \\
5 & 4413 & 75 & 48 & 3709 & 86 \\
$6 \mathrm{a}$ & 2540 & 78 & $49 \mathrm{~b}$ & 4695 & 46 \\
7 & 3464 & 72 & $50 \mathrm{a}$ & 6576 & 0 \\
8 & 5459 & 25 & 52 & 3722 & 84 \\
11 & 3497 & 63 & $54 \mathrm{a}$ & 5768 & 0 \\
12 & 4467 & 82 & 56 & 3746 & 53 \\
13 & 2606 & 86 & $57 \mathrm{a}$ & 6625 & 0 \\
14 & 1083 & 85 & $60 \mathrm{a}$ & 4757 & 28 \\
15 & 1715 & 0 & $62 \mathrm{a}$ & 5829 & 42 \\
$16 \mathrm{a}$ & 8415 & 78 & 65 & 4778 & 39 \\
18 & 1759 & 80 & 66 & 2888 & 82 \\
21 & 4522 & 89 & $67 \mathrm{a}$ & 5859 & 78 \\
$22 \mathrm{a}$ & 1142 & 73 & $69 \mathrm{a}$ & 2012 & 78 \\
$24 \mathrm{~b}$ & 4543 & 0 & 70 & 4809 & 33 \\
27 & 2672 & 0 & 72 & 5884 & 78 \\
30 & 4549 & 83 & 73 & 3900 & 59 \\
31 & 6478 & 0 & $75 \mathrm{a}$ & 4888 & 0 \\
$35 \mathrm{a}$ & 1820 & 9 & $76 \mathrm{a}$ & 4902 & 78 \\
$38 \mathrm{a}$ & 3649 & 0 & $77 \mathrm{a}$ & 6811 & 81 \\
41 & 4636 & 75 & 78 & 2188 & 0 \\
43 & 5696 & 0 & $79 \mathrm{a}$ & 5050 & 0 \\
44 & 3683 & 62 & & & \\
\hline
\end{tabular}

